\title{
ATG5 cancer mutations and alternative mRNA splicing reveal a conjugation switch that regulates ATG12-ATG5-ATG16L1 complex assembly and autophagy
}

\author{
Daric J. Wible $\mathbb{D}^{1}$, Hsueh-Ping Chao', Dean G. Tang $\mathbb{1}^{2,3}$ and Shawn B. Bratton (1) ${ }^{1}$
}

\begin{abstract}
Autophagy is critical for maintaining cellular homeostasis during times of stress, and is thought to play important roles in both tumorigenesis and tumor cell survival. Formation of autophagosomes, which mediate delivery of cytoplasmic cargo to lysosomes, requires multiple autophagy-related (ATG) protein complexes, including the ATG12-ATG5ATG16L1 complex. Herein, we report that a molecular ATG5 "conjugation switch", comprised of competing ATG12 and ubiquitin conjugation reactions, integrates ATG12-ATG5-ATG16L1 complex assembly with protein quality control of its otherwise highly unstable subunits. This conjugation switch is tightly regulated by ATG16L1, which binds to free ATG5 and mutually protects both proteins from ubiquitin conjugation and proteasomal degradation, thereby instead promoting the irreversible conjugation of ATG12 to ATG5. The resulting ATG12-ATG5 conjugate, in turn, displays enhanced affinity for ATG16L1 and thus fully stabilizes the ATG12-ATG5-ATG16L1 complex. Most importantly, we find in multiple tumor types that ATG5 somatic mutations and alternative mRNA splicing specifically disrupt the ATG16L1binding pocket in ATG5 and impair the essential ATG5-ATG16L1 interactions that are initially required for ATG12-ATG5 conjugation. Finally, we provide evidence that ATG16L2, which is overexpressed in several cancers relative to ATG16L1, hijacks the conjugation switch by competing with ATG16L1 for binding to ATG5. While ATG16L2 stabilizes ATG5 and enables ATG12-ATG5 conjugation, this endogenous dominant-negative inhibitor simultaneously displaces ATG16L1, resulting in its proteasomal degradation and a block in autophagy. Thus, collectively, our findings provide novel insights into ATG12-ATG5-ATG16L1 complex assembly and reveal multiple mechanisms wherein dysregulation of the ATG5 conjugation switch inhibits autophagy.
\end{abstract}

\section{Introduction}

Macroautophagy (hereafter referred to as autophagy) is a highly conserved catabolic process that maintains cellular homeostasis by targeting excess or damaged organelles, large protein aggregates, invading pathogens, and nonselective portions of the cytoplasm for lysosomal

Correspondence: Shawn B. Bratton (sbbratton@mdanderson.org)

${ }^{1}$ Department of Epigenetics and Molecular Carcinogenesis, The University of Texas MD Anderson Cancer Center, Smithville, TX 78957, USA

${ }^{2}$ Department of Pharmacology and Therapeutics, Roswell Park Cancer Institute, Buffalo, NY 14263, USA

Full list of author information is available at the end of the article. degradation via double-membrane vesicles, termed autophagosomes. Initially, a cup-shaped precursor membrane, or phagophore, elongates and envelops cytoplasmic cargo before closing to form a mature autophagosome, which in turn fuses with lysosomes whereupon the inner autophagosomal membrane and sequestered material are degraded by acid hydrolases ${ }^{1}$. Formation of autophagosomes requires two ubiquitin-like conjugation reactions involving autophagy-related 12 (ATG12) and members of the microtubule-associated protein 1 light chain 3 (LC3) family (homologs of yeast Atg8). Like ubiquitin, glycine residues at the immediate $\mathrm{C}$-termini of ATG12 and LC3

\section{(c) The Author(s) 2019}

(c) Open Access This article is licensed under a Creative Commons Attribution 4.0 International License, which permits use, sharing, adaptation, distribution and reproduction cc) in any medium or format, as long as you give appropriate credit to the original author(s) and the source, provide a link to the Creative Commons license, and indicate if changes were made. The images or other third party material in this article are included in the article's Creative Commons license, unless indicated otherwise in a credit line to the material. If material is not included in the article's Creative Commons license and your intended use is not permitted by statutory regulation or exceeds the permitted use, you will need to obtain permission directly from the copyright holder. To view a copy of this license, visit http://creativecommons.org/licenses/by/4.0/. 
family proteins, the latter of which are exposed following cleavage by ATG4 cysteine proteases, are first activated by the E1-like enzyme, ATG7, and then transferred to E2like enzymes, ATG10 and ATG3, respectively ${ }^{2,3}$. The ATG12 ATG10 intermediate, which possesses a highenergy thioester bond, subsequently binds to ATG5 and, independently of any known E3-like enzyme, facilitates the conjugation of ATG12 to ATG5 at Lys-130 ${ }^{4}$.

As there are no known deconjugation enzymes, ATG12-ATG5 conjugates are thought to be irreversibly formed and recruited to phagophores by dimeric ATG16L1, in conjunction with its binding partners, WD repeat domain phosphoinositide-interacting protein 2 (WIPI2) and/or RB1 inducible coiled-coil 1 (RB1CCI, also known as FIP200), thereby forming the heteromeric ATG12-ATG5-ATG16L1 complex ${ }^{3,5-7}$. LC3 ATG3 intermediates are recruited to phagophores through a direct interaction with ATG12 and/or through a membrane curvature-sensing domain in $\mathrm{ATG}^{8-11}$. The ATG12-ATG5-ATG16L1 complex subsequently acts as an E3-like enzyme to catalyze the conjugation of LC3 to phosphatidylethanolamine (PE) on the phagophore membrane ${ }^{12}$. PE-conjugated LC3 family proteins recruit diverse cytoplasmic cargoes to phagophores, either directly or through adapter proteins such as p62 (also known as SQSTM1) ${ }^{13}$. They are also implicated in the recruitment of the unc-51 like autophagy activating kinase 1 (ULK1) complex and the expansion and closure of phagophores ${ }^{14}$, as well as the fusion of mature autophagosomes with lysosomes ${ }^{15}$. While the basic ATG12 and LC3 ubiquitin-like conjugation reactions have been characterized, the mechanisms regulating ATG12 conjugation and ATG12-ATG5-ATG16L1 complex assembly remain unclear.

Preclinical data from multiple Atg gene knockout mouse models suggest that, in addition to other physiological roles, autophagy suppresses malignant transformation of normal cells, at least in part, through the degradation of oncogenic proteins, damaged mitochondria, and protein aggregates $^{16,17}$. Following transformation, however, autophagy is conversely thought to promote malignant cell survival in response to stressors found in the tumor microenvironment (e.g., nutrient deprivation, and hypoxia), thus supporting tumor growth, invasion, and metastasis, as well as diminishing the effectiveness of chemo- and radiotherapies ${ }^{18,19}$. Whether autophagy functions similarly during human tumorigenesis is still under investigation; however, multiple clinical trials are underway to evaluate the efficacy of using the lysosomotropic alkalinizing agent, hydroxychloroquine, to sensitize tumors to chemotherapy ${ }^{19}$. Seemingly in support of the proposed role of autophagy in tumor survival, core ATG genes are generally not mutated or transcriptionally downregulated in most human cancers ${ }^{20}$. However, we have discovered that ATG5 is selectively inactivated in some human tumors by somatic mutations and aberrant mRNA splicing, as well as relative overexpression of $A T G 16 L 2$, a heretofore unrecognized dominant-negative inhibitor of the ATG12-ATG5-ATG16L1 complex. Collectively, these perturbations disrupt a critical ATG5ATG16L1 interaction and elicit proteasome-dependent degradation of ATG5, ATG12, and ATG16L1. Mechanistically, we have determined that an initial ATG5ATG16L1 interaction is normally required to prevent the ubiquitin conjugation of ATG5 and instead facilitate its conjugation to ATG12, which in turn enhances its affinity for ATG16L1 and further stabilizes the ATG12-ATG5-ATG16L1 complex. Thus, the competing ubiquitin and ATG12 conjugation reactions naturally form a molecular "conjugation switch" that integrates protein quality control (PQC) of "orphaned" complex subunits with their assembly into ATG12-ATG5ATG16L1 complexes. Dysregulation of this conjugation switch through multiple mechanisms in human tumors highlights the importance of ATG5 as a critical regulator of autophagy, and is consistent with the purported role of autophagy as a suppressor of tumor initiation.

\section{Results}

An ATG5 splice site mutation in DU145 cells results in the loss of ATG5 expression and triggers proteasomal degradation of ATG12 and ATG16L1

While assessing basal autophagic flux in classical prostate cancer (PCa) cell lines, we found that DU145 cells had strikingly higher basal levels of p62 compared to LNCaP and PC-3 cells (Fig. 1a). Moreover, inhibition of autophagic flux with Bafilomycin A1 (Baf A1) treatment triggered a significant build-up of lipid-conjugated LC3B (LC3B-II) in both LNCaP and PC-3 cells, whereas no LC3B-II was detected in DU145 cells (Fig. 1a). Expression levels of ULK1 and Beclin 1-phosphatidylinositol 3-kinase catalytic subunit type 3 (PIK3C3) complex subunits were comparable between LNCaP, PC-3, and DU145 cells; however, subunits of the ATG12-ATG5-ATG16L1 complex were expressed at lower levels in LNCaP cells, and were entirely absent in DU145 cells (Fig. 1b). Treatment of DU145 cells with the proteasome inhibitor, MG132, had no effect on ATG5 expression, but triggered a buildup of both ATG16L1 and unconjugated ATG12 (Fig. 1c, lanes 5 and 6), suggesting that DU145 cells did not express ATG5, and that orphaned ATG12 and ATG16L1 underwent proteasomal degradation.

To determine whether ATG12 and ATG16L1 stabilities were dependent upon ATG5, we knocked out ATG5 in LNCaP and PC-3 cells using CRISPR/Cas9, which led to the loss of both ATG12 and ATG16L1 expression (Fig. 1d, lanes 3 and 7). Consistent with the previously reported compensatory induction of autophagy in response to 


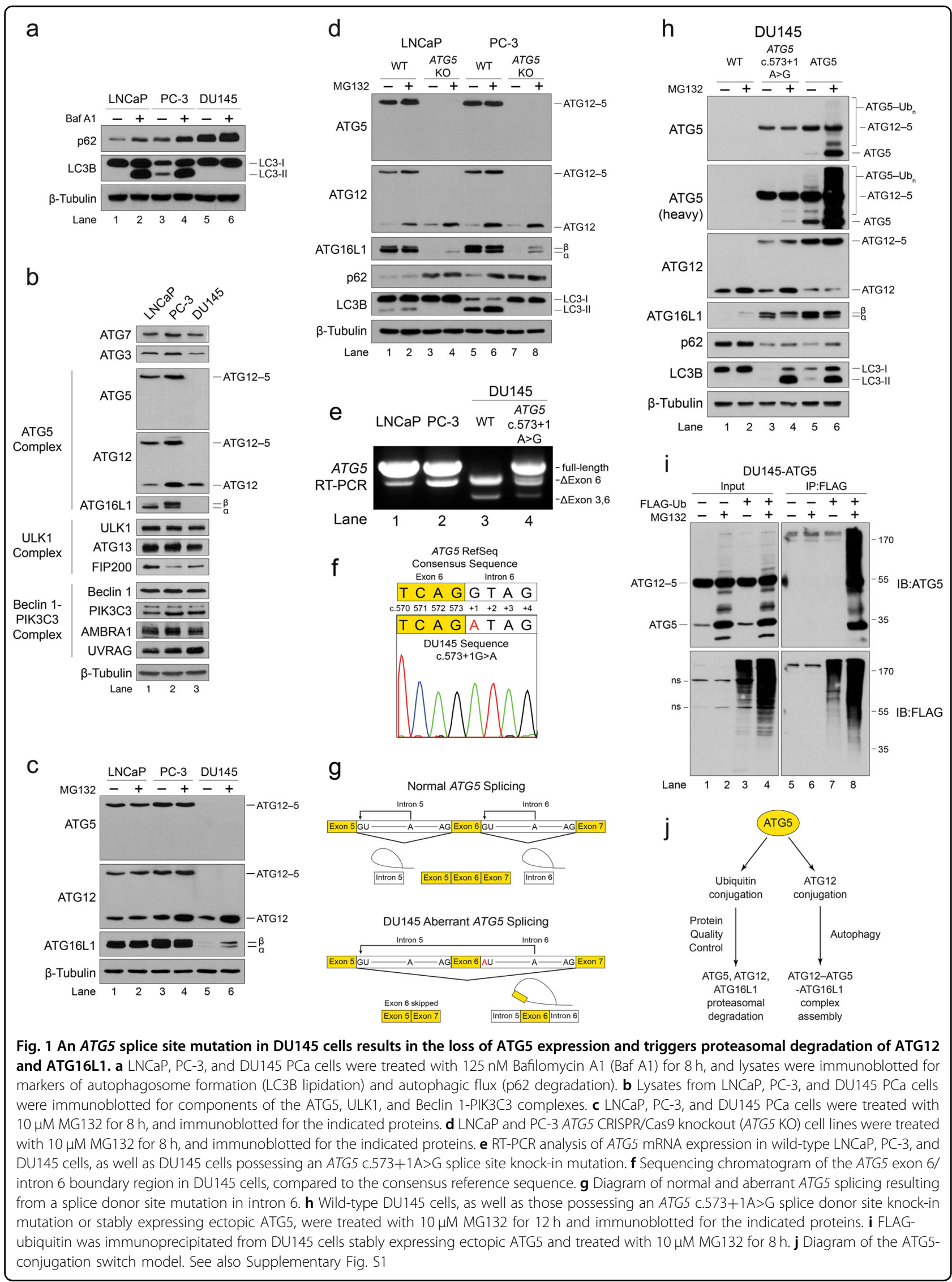


proteasome inhibition ${ }^{21}$, MG132 treatment triggered an increase in LC3B-II levels in wild-type LNCaP and PC-3 cells (Fig. 1d, lanes 1, 2, 5, and 6), whereas no LC3B-II was detected in ATG5 KO cells (Fig. 1d, lanes 3, 4, 7, and 8). MG132 treatment did, however, partially rescue ATG16L1 and unconjugated ATG12 expression in ATG5 KO cells (Fig. 1d, lanes 4 and 8). ATG12 and ATG16L1 were similarly lost in $\operatorname{Atg} 5^{-1-}$ mouse embryonic fibroblasts (MEFs) (Supplementary Fig. S1, lane 3), confirming that ATG12 and ATG16L1 stabilities were dependent upon ATG5 in multiple cell types.

In an effort to understand why DU145 cells lacked ATG5, we noted a recent study in which DU145 cells were found to undergo extensive alternative mRNA splicing for unknown reasons ${ }^{22}$. We confirmed the absence of full-length ATG5 mRNA expression and, instead, found two splice variants that excluded either exon 6 or both exons 3 and 6 (Fig. 1e, lane 3). Since DU145 cells reportedly possess a hemizygous $6 \mathrm{q}$ deletion encompassing the ATG5 locus ${ }^{23}$, and given that both mRNA variants lacked exon 6 , we speculated that a splice site mutation proximate to exon 6 in the remaining allele might be responsible for "exon skipping" and the complete absence of full-length ATG5 mRNA. We, therefore, sequenced the splice sites flanking exon 6 and discovered a mutation (c.573+1G $>$ A) at the most $5^{\prime}$ nucleotide of intron 6 (Fig. 1f). This mutation affected the conserved splice donor site and was predicted to prevent nucleophilic attack by the 2 '-hydroxyl group of the branch site adenosine, thereby triggering attack at the next upstream splice donor site of intron 5 and aberrant excision of exon 6 along with introns 5 and 6 (Fig. 1g). Remarkably, reverting the mutant splice site allele to wild type in DU145 cells using CRISPR/Cas9 rescued full-length ATG5 mRNA expression (Fig. 1e, lane 4), as well as ATG12-ATG5 conjugation, functional ATG12-ATG5-ATG16L1 complex formation and autophagic flux, as determined by LC3 lipidation and p62 degradation (Fig. 1h, lanes 3 and 4). Collectively, these data proved that the autophagic defect in DU145 cells was caused by an ATG5 splice donor site mutation, which resulted in the loss of ATG5 expression and proteasomal degradation of orphaned ATG12 and ATG16L1.

While reversion of the ATG5 splice site mutation in DU145 cells restored the formation of ATG12-ATG5 conjugates, unconjugated ATG5 still remained undetectable (Fig. 1h, lane 3). Unconjugated ATG5 was faintly rescued by a $12 \mathrm{~h}$ incubation with MG132; however, this had no effect on conjugated ATG12-ATG5 (Fig. 1h, lane 4), suggesting that, like orphaned ATG12 and ATG16L1, free ATG5 also underwent proteasomal degradation in DU145 cells. Proteasomal degradation of free ATG5 is consistent with the fact that unconjugated ATG5 was also undetectable in wild-type $\mathrm{LNCaP}$ and $\mathrm{PC}-3$ cells, as well as MEFs (Fig. 1b, lanes 1 and 2; Supplementary Fig. S1, lane 1). To confirm, we treated DU145 cells stably expressing ectopic ATG5 with MG132, which triggered a dramatic accumulation of ATG5 and a ladder of highmolecular weight species indicative of polyubiquitination (Fig. 1h, lanes 5 and 6). Similar results were obtained in Atg5 $5^{-1-}$ MEFs stably expressing ectopic murine ATG5 (Supplementary Fig. S1, lanes 7 and 8). Coimmunoprecipitation of ATG5 using FLAG-tagged ubiquitin confirmed that free ATG5 was polyubiquitinated (Fig. 1i). Together, these data support the hypothesis that ATG5, ATG12, and ATG16L1 all undergo PQC when orphaned from the intact ATG12-ATG5-ATG16L1 complex. Since ATG5 is targeted by both ubiquitin and ATG12 conjugation reactions, we proposed a model in which the competing conjugation reactions effectively function as a molecular "conjugation switch" that regulates autophagy by promoting either the proteasomal degradation of ATG5, or its assembly into stable ATG12-ATG5-ATG16L1 complexes (Fig. 1j).

\section{Somatic ATG5 splice site mutations and alternative mRNA splicing prevent ATG12 conjugation and trigger PQC of unstable protein isoforms}

Having identified an ATG5 splice site mutation in DU145 PCa cells that resulted in the loss of ATG5, ATG12, and ATG16L1 protein expression and the inactivation of autophagy, we next questioned whether other tumors might possess similar splice site mutations that impair mRNA splicing of ATG5. We searched human tumor mutation databases and found several unique ATG5 splice site mutations identified in multiple tumor types. All but one of these mutations were predicted, in silico $^{24}$, to either destroy the consensus splice site and trigger exon skipping or activate a cryptic splice site that would introduce a frameshift and prevent expression of full-length ATG5 (Fig. 2a). However, it remained unclear whether these predicted splicing defects would lead to the loss of functional ATG5 expression as we had observed in DU145 cells.

To test whether known ATG5 mRNA splice variants, listed in the National Center for Biotechnology Information (NCBI) RefSeq database ${ }^{25}$, encoded functional protein isoforms (Fig. 2b; Supplementary Fig. S2a, b), we stably expressed HA-tagged versions of each predicted isoform in DU145 cells. Notably, only full-length ATG5 (isoform a) formed functional ATG12-ATG5-ATG16L1 complexes as determined by the rescue of conjugated ATG12-ATG5 and ATG16L1 expression, as well as LC3B lipidation and p62 degradation (Fig. 2c, lanes 3 and 4). All other isoforms were essentially undetectable in the absence of proteasomal inhibition, which caused a dramatic accumulation of their polyubiquitinated forms (Fig. 2c, lanes 5-14). Importantly, polyubiquitination and 


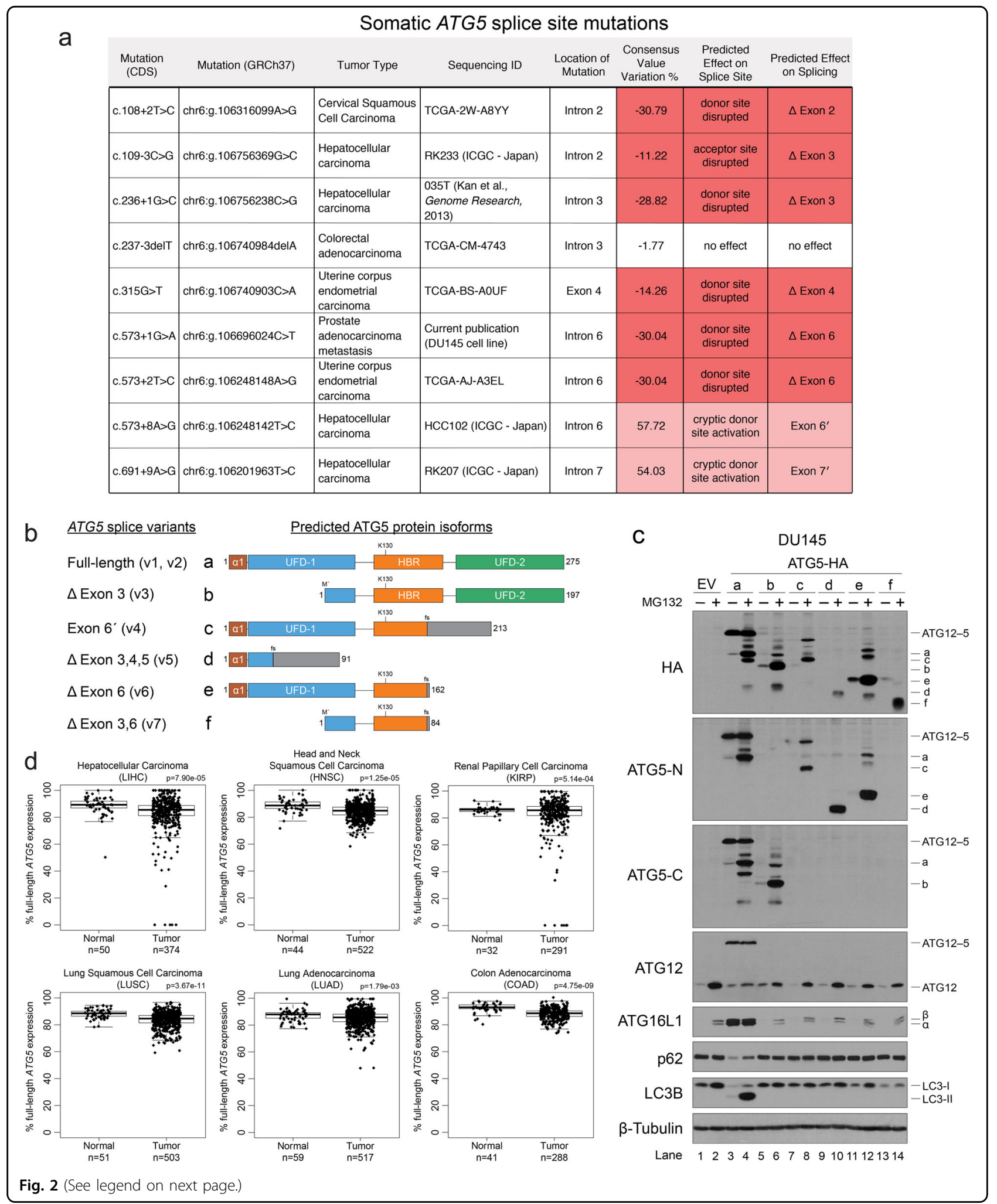

proteasomal degradation occurred with isoforms containing C-terminal truncations caused by frameshifts and premature stop codons (isoforms $\mathrm{c}-\mathrm{f}$ ), as well as those with N-terminal deletions, predicted to result from the introduction of alternative translation initiation sites (isoforms b and f) (Fig. 2b; Supplementary Fig. S2a, b). 
(see figure on previous page)

Fig. 2 Somatic ATG5 splice mutations and alternative mRNA splicing prevent ATG12 conjugation and trigger PQC of highly unstable protein isoforms. a Table of somatic ATG5 splice site mutations identified in human tumor samples and cancer cell lines. The specific DNA mutations are indicated based on the coding sequence (CDS) and the genomic sequence (GRCh37). The location of the mutations within splice acceptor or donor sites of specific introns/exons is shown, as is the tumor type and sequencing ID of the tumors in which the mutations were identified. Finally, the effect of splice site mutations on ATG5 splicing was predicted, in silico, using the Human Splicing Finder (http://www.umd.be/ $\mathrm{HSF} /)^{24}$. The consensus value (CV) variation \% is the difference between wild-type and mutant CVs as a percentage of the total wild-type CV. CVs are calculated based on positional variation from the consensus splicing motif. Predicted skipped exons are indicated with $(\Delta)$ and predicted cryptic exons are indicated with ('). b Diagram of predicted isoforms encoded by known ATG5 splice variants. UFD-1 and UFD-2 refer to the ubiquitin-fold domains; HBR refers to the helix-bundle region; and a1 refers to the alpha-1 helix domain ${ }^{27}$. c DU145 cells, stably expressing an empty vector (EV) or HA-tagged ATG5 alternative protein isoforms, were treated with $10 \mu \mathrm{M} \mathrm{MG132}$ for $8 \mathrm{~h}$ and immunoblotted for the indicated proteins. ATG5-N and ATG5-C refer to antibodies raised to the $\mathrm{N}$ - or C-terminus of ATG5, respectively. $\mathbf{d}$ Tukey box plots indicating the percentage of full-length ATG5 mRNA (ATG5V7; uC003prf) expression to total ATG5 mRNA expression from normal and tumor tissues. See also Supplementary Fig. S2

Thus, both the N-and C-termini of ATG5 were critical for its stability. Moreover, no single region of ATG5 was shared among all isoforms, suggesting that ATG5 may be polyubiquitinated by multiple E3 ubiquitin ligases that recognize nonoverlapping regions of the protein.

The fact that all ATG5 protein isoforms encoded by known splice variants failed to conjugate with ATG12and instead underwent ubiquitin conjugation that targeted them for $\mathrm{PQC}-$ suggested that any somatic splice site mutation that disrupts normal ATG5 mRNA splicing in tumors likely leads to a complete loss of ATG5 function. These findings also raised the possibility that, independently of splice site mutations, tumor cells may routinely utilize "conventional" alternative splicing of ATG5 as a mechanism to reversibly regulate ATG12-ATG5-ATG16L1 complex formation and autophagy. As aberrant mRNA splicing frequently occurs in tumors ${ }^{26}$, we tested this hypothesis by comparing the percentage of full-length ATG5 (ATG5v1) expression from total $A T G 5$ expression in normal and tumor samples within The Cancer Genome Atlas (TCGA) datasets possessing at least 15 normal samples. While full-length $A T G 5 v 1$ was the dominant species in all tumor types, we found a statistically significant reduction in the percentage of full-length ATG5 expression in 6 out of 12 tumor types ( $p<0.01$; Fig. 2 d). Prostate adenocarcinoma was the lone tumor type in which the average percentage of fulllength ATG5v1 expression was increased in tumors compared to normal tissue; however, there were also a number of outlier prostate tumor samples in which fulllength $A T G 5 v 1$ expression was dramatically reduced or completely lost (Supplementary Fig. S2c). Collectively, these data suggest that ATG12-ATG5-ATG16L1 complex assembly is regulated in tumors by conventional alternative ATG5 mRNA splicing, as well as aberrant splicing resulting from ATG5 splice site mutations, both of which lead to the coordinated degradation of ATG5, ATG12, and ATG16L1.

\section{Somatic ATG5 missense, nonsense, and deletion mutations trigger PQC by directly disrupting the ATG16L1-binding pocket in ATG5}

The fact that every alternative ATG5 isoform failed to conjugate to ATG12 was somewhat surprising given that all but one retained Lys-130 and were theoretically capable of conjugating to ATG12 (Fig. 2b). It was, therefore, unclear if the turnover of these ATG5 isoforms resulted from inherent instability due to improper folding, their inability to conjugate to ATG12, or perhaps because the $\mathrm{N}$ and/or C-terminal deletions disrupted other interactions that were essential for ATG5 stability. In the ATG12 $(\Delta \mathrm{N}$ terminus)-ATG5-ATG16L1 (N-terminus) crystal structure, $\mathrm{N}$ - and C-terminal residues of ATG5 are located at the ATG16L1-binding interface, as opposed to the ATG12-binding interface ${ }^{27}$. Consequently, we suspected that the stability of ATG5, and thus its ability to conjugate to ATG12, might depend upon its initial interaction with ATG16L1. Importantly, this prediction differed from the current model of ATG12-ATG5-ATG16L1 complex formation in which conjugated ATG12-ATG5, constitutively expressed in the cytoplasm, is proposed to be recruited to phagophores by membrane-bound ATG16L $1^{2,3}$.

To test this hypothesis, we used the ATG12 $\Delta$ N-ATG5ATG16L1N crystal structure as a guide to introduce mutations that would be predicted to disrupt the ATG5ATG16L1 interaction ${ }^{27}$. Stable expression of these ATG5 mutants in naturally deficient DU145 cells revealed several key residues (highlighted in magenta) that were essential for ATG5, ATG12, and ATG16L1 stability, ATG12 conjugation, and functional ATG12-ATG5ATG16L1 complex formation, as determined by LC3B lipidation and p62 degradation (Fig. 3a). The most effective mutations (G84S, D88A, and I240S) led to complete degradation of the mutant protein, which fully prevented LC3B lipidation and caused an accumulation of p62 that was indicative of a block in autophagic flux (Fig. 3a, lanes 


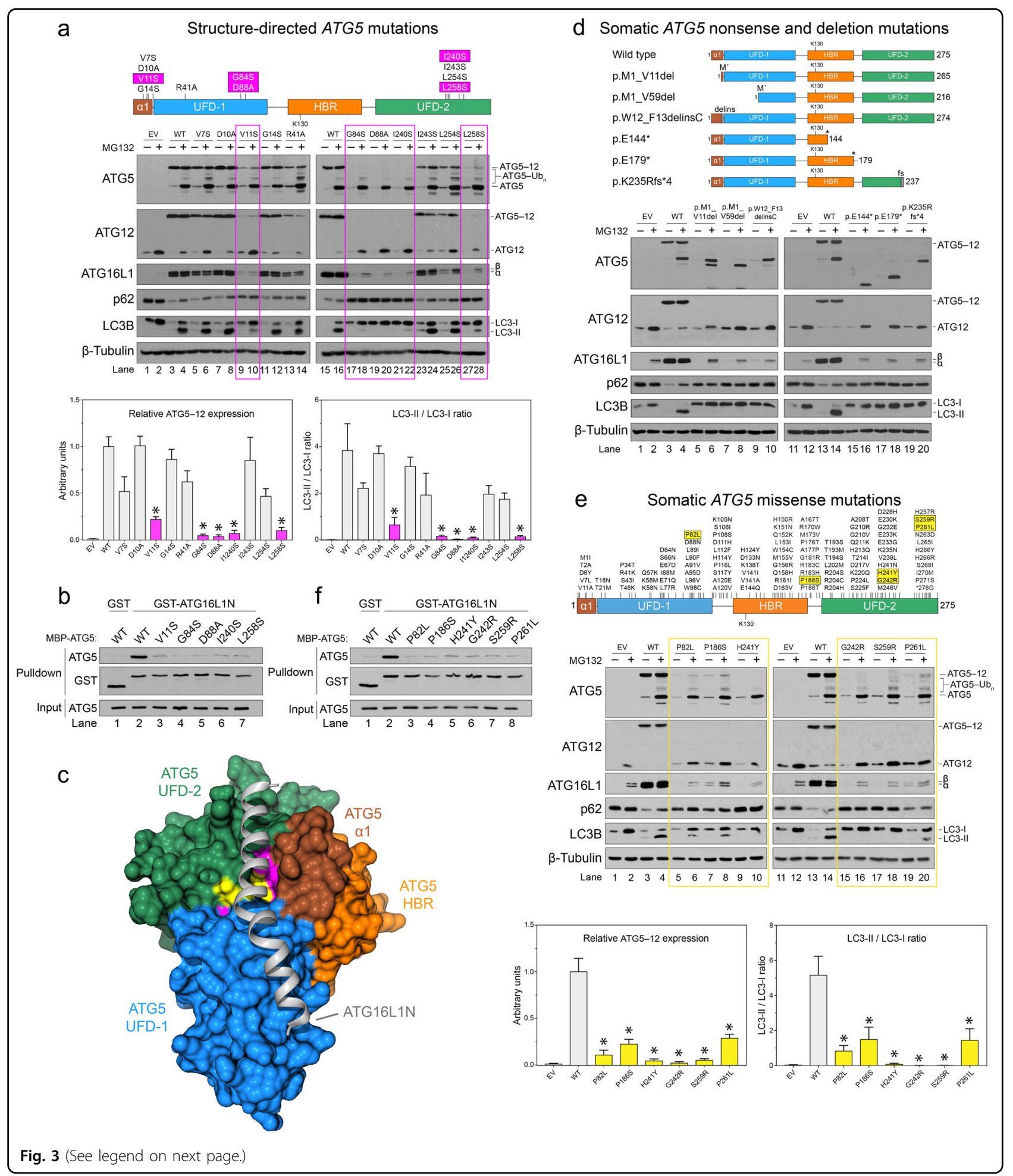

17-22). Using a glutathione $S$-transferase (GST) pulldown assay with bacterially expressed and purified GSTATG16L1N and His-tagged wild-type and mutant ATG5 proteins, we confirmed that these mutations, along with the similarly effective mutations, V11S and L258S, disrupted the interaction of ATG16L1N with ATG5, in vitro (Fig. 3b). Mapping the loss-of-function mutations onto a surface rendering of the ATG12 $\Delta \mathrm{N}-\mathrm{ATG} 5-\mathrm{ATG} 16 \mathrm{~L} 1 \mathrm{~N}$ 
(see figure on previous page)

Fig. 3 Somatic ATG5 missense, nonsense, and deletion mutations trigger PQC by directly disrupting the ATG16L1-binding pocket of ATG5. a DU145 cells, stably expressing empty vector (EV), wild-type ATG5 (WT), or ATG5 mutants designed to disrupt the ATG16L1-binding region, were treated with $10 \mu \mathrm{M} \mathrm{MG132}$ for $8 \mathrm{~h}$ and immunoblotted for the indicated proteins. Mutations that dramatically impair ATG12 and LC3B conjugation reactions are highlighted with magenta. The ATG12-ATG5 conjugate expression levels and LC3-II/LC3-I ratios from MG132 treated cells were quantified and graphed below (*, $p<0.01$ ). b GST pull downs were performed using GST-ATG16L1N and recombinant wild-type or mutant ATG5. c Rendering of the ATG12 $\triangle N$-ATG5-ATG16L1N crystal structure (PDB ID: 4GDL). The ribbon backbone of ATG16L1N is depicted in gray. The surface rendering of ATG5 includes the a1-helix (brown), UFD-1 (blue), HBR (orange), and UFD-2 (green) domains. ATG12 is not visible in this orientation. Critical residues identified from targeted structural analyses are highlighted in magenta, while those arising from somatic missense mutations identified in tumors are highlighted in yellow. $\mathbf{d}$ Diagram of proteins predicted to result from ATG5 nonsense and deletion mutations, and their fate upon expression in DU145 cells. e Diagram of ATG5 missense mutations identified in human tumors and their fate upon expression in DU145 cells. The ATG12-ATG5 conjugate expression levels and LC3-II/LC3-I ratios from MG132 treated cells were quantified and graphed below $\left.{ }^{*}, p<0.01\right)$. Note the missense mutations (highlighted in yellow) that dramatically impair ATG12 conjugation and LC3B conjugation. $\mathbf{f}$ GST pull downs were performed using GST-ATG16L1N and recombinant wild-type or mutant ATG5. See also Supplementary Fig. S3 and Supplementary Table S1

structure revealed a critical binding pocket where the $\mathrm{N}$ terminal $\alpha 1$-helix and UFD-1 domains converged with the C-terminal UFD-2 domain (Fig. 3c, magenta). The fact that this ATG16L1-binding pocket was comprised of residues at both the $\mathrm{N}$ and $\mathrm{C}$-terminal regions of ATG5 explained why all of the alternative protein isoforms containing $\mathrm{N}$ and/or C-terminal deletions were completely destabilized and failed to undergo ATG12 conjugation (Fig. 2b, c).

To determine if somatic mutations identified in human tumor samples directly affected this critical interaction between ATG5 and ATG16L1, we next compiled a list of all somatic ATG5 coding-sequence mutations that have been identified across a multitude of human tumors and cancer cell lines (Supplementary Table S1). Notably, a recurrent frameshift mutation (c.704delA), predicted to cause a 38 amino acid C-terminal truncation (p. K235Rfs*4), has been identified in 17 unique tumors and cancer cell lines, including LNCaP PCa cells (Fig. 3d; Supplementary Table S1). Stable expression of this truncated mutant in DU145 cells, as well as five others predicted to arise from other ATG5 nonsense or deletion mutations, failed to rescue the autophagy defect and resulted in complete degradation of the mutant proteins, along with ATG12 and ATG16L1 (Fig. 3d). Remarkably, even very small alterations to the $\mathrm{N}$ - or C-termini, including c.26_38delGTT (p.W12_F13delinsC), which affects only two amino acids within the $\mathrm{N}$-terminal $\alpha 1$ helix domain, completely destabilized ATG5 (Fig. 3d, lanes 9 and 10). We, therefore, classified all somatic ATG5 nonsense and deletion mutations affecting the $\mathrm{N}$ - or $\mathrm{C}$ terminus as amorphic, loss-of-function mutations (Supplementary Table S1).

We also stably expressed more than 30 ATG5 missense mutants in DU145 cells and found several (highlighted in yellow) that resulted in proteasomal degradation of ATG5, ATG12, and ATG16L1, and impaired or completely eliminated LC3B lipidation and p62 degradation
(Fig. 3e; Supplementary Fig. S3a and b). H241Y, G242R, and S259R mutations were fully penetrant and thus were also classified as amorphic, loss-of-function mutations (Fig. 3e, lanes 9, 10, and 15-18; Supplementary Table S1). Interestingly, these residues were located directly adjacent to those we had determined were essential for ATG5 stability and ATG12 conjugation (Fig. 3c, note the proximity of yellow and magenta-colored residues). Since P82L, P186S, and P261L mutations all caused significant, albeit partial, impairment of ATG12 conjugation, we classified these as hypomorphic, partial loss-of-function mutations (Fig. 3e, lanes 5-8, 19, and 20; Supplementary Table S1). These proline residues are not found on the surface of ATG5, but localize deeper within the protein core and are therefore likely to be important for proper conformation of the critical $\mathrm{N}$ - and C-terminal domains. Regardless, as before, each of these amorphic or hypomorphic mutants failed to bind ATG16L1 in vitro (Fig. 3f). Based on these results, we would expect other mutations that affect the ATG16L1-binding pocket, including p. R9_D10delinsH, p.D88N, p.P186T, and p.M246V (Supplementary Table S1), to similarly impair or completely block ATG12-ATG5-ATG16L1 complex formation and autophagy. Collectively, our functional analysis of somatic ATG5 mutations in human tumors demonstrated that a single recurring somatic frameshift mutation (c.704delA), as well as a variety of other unique somatic mutations specifically disrupt the ATG16L1-binding pocket. This effectively "flips" the ATG5 conjugation switch from ATG12 to ubiquitin conjugation, and in turn triggers PQC of ATG5, ATG12, and ATG16L1.

\section{ATG12 conjugation to ATG5 enhances its affinity for ATG16L1 and stabilizes otherwise transient ATG5-ATG16L1 interactions}

Contrary to the current model of ATG12-ATG5ATG16L1 complex formation, in which ATG12 is conjugated to ATG5 prior to its recruitment to the 
phagophore by ATG16L1 $1^{2,3}$, our characterization of ATG5 mutations strongly suggested that the interaction of ATG5 with ATG16L1 was a prerequisite for ATG12 conjugation. To further evaluate this model, we stably expressed an ATG5 fusion protein in DU145 cells, in which an N-terminal fragment of ATG16L1 (aa 11-36), corresponding to its ATG5-binding domain, was tethered to ATG5 via a flexible linker (16L1N-ATG5). Remarkably, the tethered $16 \mathrm{~L} 1 \mathrm{~N}$ fragment almost entirely prevented the ubiquitination and turnover of free ATG5 (Fig. 4a, compare unconjugated ATG5 in lanes 3 and 5). The tethered $16 \mathrm{~L} 1 \mathrm{~N}$ fragment was even able to partially stabilize the otherwise unstable ATG5 (D88A) mutant and allow it to undergo ATG12 conjugation (Fig. 3a, lanes 19 and 20; Supplementary Fig. S4a, lanes 7 and 8), thereby confirming that mutation of the ATG16L1-binding pocket did not prevent ATG12 conjugation due to protein misfolding, etc. To ensure that binding of the tethered $16 \mathrm{~L} 1 \mathrm{~N}$ fragment was responsible for the stabilization of ATG5, we also mutated the tethered 16L1N fragment (I17W), along with the ATG16L1-binding pocket in ATG5 (D88A), and found that disruption of this specific interaction resulted in complete degradation of the fusion protein (Supplementary Fig. S4a, compare 16L1N-ATG5 in lanes 5, 7, and 9).

In addition to stabilizing the ATG5 fusion protein and facilitating its conjugation to ATG12, the tethered $16 \mathrm{~L} 1 \mathrm{~N}$ fragment also prevented the fusion protein from binding to and stabilizing endogenous ATG16L1, resulting in its proteasomal degradation and a block in LC3B lipidation and p62 degradation (Fig. 4a, lanes 3-6). This indicated that endogenous ATG16L1 possessed essential autophagic functions beyond simply stabilizing ATG5, which is consistent with the proposed role for its $\mathrm{C}$-terminus in localizing the ATG12-ATG5-ATG16L1 complex to phagophores through its interactions with WIPI2 and/or FIP200 ${ }^{5-7}$. Interestingly, we also stably expressed fulllength ATG16L1 in DU145 cells, as well as a truncated ATG16L1 that lacked the N-terminal ATG5-binding domain $(16 \mathrm{~L} 1 \Delta \mathrm{N})$, and found that, in the absence of ATG5, proteasomal degradation of ectopically expressed ATG16L1 was wholly prevented by removal of its $\mathrm{N}$ terminus (Fig. 4a, lanes 7-10). Thus, in addition to binding ATG5 and protecting it from ubiquitination and proteasomal degradation, the N-terminus of ATG16L1 also functioned as a degron that targeted ATG16L1 for PQC when orphaned from ATG5.

While these findings suggested that ATG5 and ATG16L1 mutually stabilized and protected one another from PQC, they did not address the role of ATG12 conjugation on ATG5 or ATG16L1 stability. To investigate this, we stably expressed a K130R mutant of ATG5, which cannot undergo ATG12 conjugation, in DU145 cells and found that it was less stable than wild-type conjugated
ATG5 and consequently stabilized ATG16L1 less efficiently (Fig. 4b, compare lanes 3 and 5). We also blocked ATG12 conjugation in LNCaP and PC-3 cells by knocking out ATG7 using CRISPR/Cas9 and found that it substantially reduced ATG5 and ATG16L1 expression levels (Supplementary Fig. S4b, compare lanes 1, 3, 5, and 7). ATG5 and ATG16L1 levels were similarly reduced in $\operatorname{Atg} 7^{-1}$ and $\operatorname{Atg} 12^{-/-}$MEFs (Supplementary Fig. S4c, lanes 1-4). A cycloheximide chase experiment confirmed that free ATG5 (and ATG16L1) possessed dramatically shorter half-lives in ATG7 KO PC-3 cells compared to conjugated ATG5 in wild-type cells (Fig. 4c). Together, these results suggested that the initial ATG5-ATG16L1 interaction was transient, perhaps due to lower affinity, or that the ATG12-ATG5 conjugate was resistant to ubiquitination.

The fact that ATG5-K130R was still readily polyubiquitinated and degraded (Fig. 4b, lanes 5 and 6) suggested that ATG12 conjugation did not stabilize ATG5 simply by blocking ubiquitination at Lys-130 (although, importantly, ubiquitination at Lys-130 would prevent conjugation of ATG12 to ATG5). Instead, since tethering of the ATG16L1N fragment to ATG5 was sufficient to fully stabilize unconjugated ATG5 (Fig. 4a, lane 5 and 6), we speculated that ATG12 conjugation induced a conformational change in ATG5 that increased its overall affinity for ATG16L1 and further stabilized the complex. Despite the high degree of structural similarity of ATG5 within the conjugated ATG12 $\triangle \mathrm{N}-\mathrm{ATG} 5$ ATG16L1N and unconjugated ATG5-ATG16L1N crystal structures (Supplementary Fig. S4d) ${ }^{27,28}$, we indeed found that conjugated ATG12-ATG5 possessed a roughly tenfold higher affinity for GST-ATG16L1N than did unconjugated ATG5 based on quantification of GST pulldown assays performed using cell lysates (Fig. 4d). While this result potentially explained the dramatic difference in stability between conjugated ATG12-ATG5 and unconjugated ATG5, it should be noted that it does not rule out the possibility that ATG12 conjugation may also directly impair ATG5 ubiquitination by sterically hindering the recruitment of E3 ubiquitin ligase(s) and/or access to key lysine residues on ATG5. It is also possible that additional unknown protein(s) associated with the native ATG12-ATG5-ATG16L1 complex (not present in the crystal structures) serve to enhance the interaction of ATG16L1 with conjugated ATG12-ATG5 and/or block E3 ligase recruitment.

\section{ATG16L2 functions as a dominant-negative inhibitor of autophagy by competing with ATG16L1 for binding to ATG5}

Having determined that disruption of the ATG16L1binding pocket in ATG5, through somatic mutations and/ or alternative mRNA splicing, prevented ATG12 


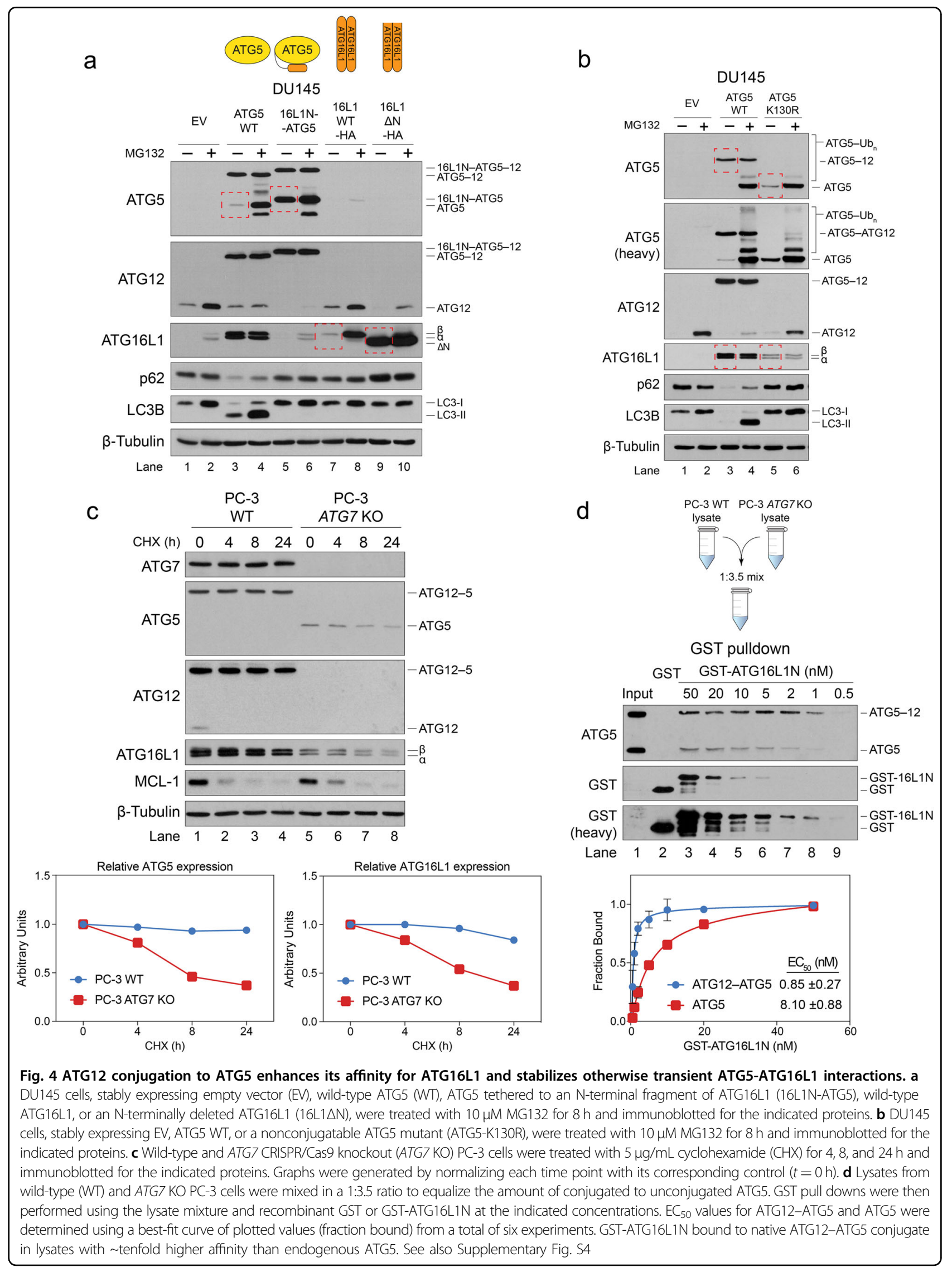


conjugation and formation of functional ATG12-ATG5ATG16L1 complexes (Figs. 2 and 3), we expected that deletion of ATG16L1 would similarly block both ATG12 and LC3 conjugation reactions. However, to our surprise, while knocking out ATG16L1 in DU145 cells stably expressing ATG5 completely blocked LC3B lipidation and p62 degradation, ATG12 conjugation still occurred, albeit at reduced levels (Fig. 5a). ATG12-ATG5 conjugation was similarly impaired, but still present, in ATG16L1 KO LNCaP and PC-3 cells (Fig. 5b). Finally, ATG12 conjugation was also observed in $\operatorname{Atg}_{16 l 1^{\Delta / \Delta}}$ MEFs (Supplementary Fig. S4c, lanes 7 and 8).

In an attempt to resolve the apparent discrepancy between the ATG5 mutants that failed to bind ATG16L1, resulting in complete turnover of the ATG12-ATG5ATG16L1 complex (Fig. 3), versus the ATG16L1-knockout data, wherein ATG12-ATG5 levels were only partially reduced (Fig. 5a, b), we postulated that ATG16L1independent ATG12 conjugation might have occurred as a result of compensation from other ATG5-interacting proteins. ATG16L2 and TECPR1 reportedly bind to the same ATG16L1-binding pocket ${ }^{28,29}$, and thus might have similarly protected ATG5 from proteasomal degradation in ATG16L1 KO cells (Supplementary Fig. S5a). Indeed, while ATG16L1 underwent complete PQC when expressed in ATG5-deficient DU145 cells, ATG16L2 and TECPR1 were both considerably more stable (Fig. 5c). Stably co-expressing ATG16L1, ATG16L2, or TECPR1 along with ATG5 in DU145 cells resulted in dramatic stabilization of unconjugated ATG5 (Fig. 5d, top panel, compare lanes $1,2,4$, and 6). This stabilization was completely reversed by either deleting or mutating the ATG5-binding domains in each protein (Fig. 5d, lanes 3, 5 , and 7 ), confirming that multiple proteins are capable of binding unconjugated ATG5 and preventing ubiquitination and proteasomal degradation. We then compared the relative affinities of GST-tagged ATG16L1N, ATG16L2N, and the TECPR1 ATG5-interacting region (TECAIR) region of TECPR $1^{28}$, for both conjugated ATG12-ATG5 and unconjugated ATG5 using GST pull-down assays. In agreement with previous biochemical characterizations of ATG16L2 and TECPR1 ${ }^{28,29}$, we did not observe obvious differences in the affinities of GST-ATG16L1N and GST-ATG16L2N for either ATG5 or ATG12-ATG5, whereas GST-TECAIR displayed lower affinities for both at $\mathrm{pH} 8.0$ (Fig. 5e). However, as we observed with GST-ATG16L1N (Fig. 4d; Fig. 5e, lanes 3-6), GST-ATG16L2N and GST-TECAIR both possessed higher affinities for conjugated ATG12-ATG5 compared to unconjugated ATG5 (Fig. 5e, lanes 7-14), indicating that the ATG12-ATG5-ATG16L2 and ATG12-ATG5-TECPR1 complexes were stabilized by ATG12 conjugation in a manner similar to that observed for the ATG12-ATG5-ATG16L1 complex.
Given these results, we then questioned whether ATG16L2 or TECPR1, which are not thought to catalyze LC3 lipidation ${ }^{28,29}$, might act as dominant-negative inhibitors of autophagy by competing with ATG16L1 for binding to ATG5 (Supplementary Fig. S5a). Stable overexpression of ATG16L2 or TECPR1 in wild-type LNCaP cells, which express lower levels of the ATG12-ATG5ATG16L1 complex due to a hemizygous loss-of-function ATG5 (c.704delA) mutation (Figs. 1b, 3d; Supplementary Table S1), triggered the displacement of endogenous ATG16L1 from ATG5, resulting in a significant loss of ATG16L1 and the inhibition of autophagy, as determined by a decrease in LC3 lipidation, with minimal effect on ATG12-ATG5 conjugation (Fig. 5f, lanes 3, 4, 7, and 8). Mutating or deleting the ATG5-binding domains of ATG16L2 and TECPR1 reversed these effects (Fig. 5f, lanes 5, 6, 9, and 10). Consistent with its higher affinity for ATG5 (Fig. 5e), ATG16L2 was more effective than TECPR1 at displacing ATG16L1 and inhibiting LC3B lipidation (Fig. 5f, lanes 3, 4, 7, and 8). Since either upregulation of ATG16L2 expression or downregulation of ATG16L1 expression in human tumors should increase the competitive binding of ATG16L2 to ATG5, we calculated the ATG16L2:ATG16L1 mRNA expression ratio in normal and tumor samples from the TCGA mRNA datasets. The ATG16L2:ATG16L1 ratios in the vast majority of normal samples were $<1.0$, suggesting that ATG16L1 was generally more highly expressed than ATG16L2. However, statistically significant increases in ATG16L2 expression were observed, relative to ATG16L1, in 5 out of the 12 tumor types analyzed ( $p<0.01$; Fig. $5 \mathrm{~g})$. Together, these data indicated that ATG16L2-and to a lesser degree TECPR1-can act as dominant-negative inhibitors of autophagy by competitively binding to ATG5 and triggering PQC of the displaced ATG16L1. Overexpression of $A T G 16 L 2$, therefore represents yet another mechanism for selectively impairing ATG12-ATG5ATG16L1 complex assembly and autophagy in tumors.

To evaluate the role of endogenous ATG16L2 and TECPR1 in ATG12-ATG5-ATG16L1 complex formation, we knocked out ATG16L2 and TECPR1 in PC-3 cells using CRISPR/Cas9. Due to the lack of specific antibodies for these proteins, we verified knockout efficiency using Sanger sequencing and the Inference of CRISPR Edits (ICE) Analysis Tool (Supplementary Fig. S5b and c). Unlike the knockout of $A T G 16 L 1$, which dramatically reduced ATG12-ATG5 levels, knockout of ATG16L2 or TECPR1 alone in PC-3 cells had no effect on ATG12-ATG5 conjugation (Fig. 5h, lanes 1-4). Given the similar affinities of ATG16L1 and ATG16L2 for ATG5 (Fig. 5e ${ }^{28,29}$, this suggested that ATG16L2 was likely expressed at lower levels than ATG16L1. However, knockout of $A T G 16 L 2$ and TECPR1, in combination with $A T G 16 L 1$, further reduced ATG12 conjugation compared 





(see figure on previous page)

Fig. 5 ATG16L2 functions as a dominant-negative inhibitor of autophagy by competing with ATG16L1 for binding to ATG5. a DU145 wildtype (WT) and ATG16L1 CRISPR/Cas9 knockout cells (ATG16L1 KO), stably expressing empty vector (EV) or ATG5, were treated with $10 \mu \mathrm{M}$ MG132 for $8 \mathrm{~h}$ and immunoblotted for the indicated proteins. b LNCaP and PC-3 ATG16L1 CRISPR/Cas9 knockout cell lines (ATG16L1 KO) were treated with $125 \mathrm{nM}$ Baf $\mathrm{A} 1$ for $8 \mathrm{~h}$ and immunoblotted for the indicated proteins. c DU145 cells, stably expressing an empty vector (EV), ATG16L1-HA, ATG16L2HA, or HA-TECPR1, were treated with $10 \mu \mathrm{M}$ MG132 for $8 \mathrm{~h}$ and immunoblotted with HA and $\beta$-tubulin antibodies. $\mathbf{d}$ Lysates from DU145 cells, stably co-expressing ATG5 and either ATG16L1-HA, ATG16L2-HA, HA-TECPR1, or their corresponding binding mutants ( $\triangle \mathrm{N}$ or I528W) were immunoblotted for ATG5, HA and $\beta$-tubulin. e Lysates from wild-type (WT) and ATG7 KO PC-3 cells were mixed in a 1:3.5 ratio to normalize the amount of conjugated to unconjugated ATG5. As in Fig. 4d, GST pull downs were performed using the lysate mixture with recombinant GST (control), GST-ATG16L1N, GSTATG16L2N, or GST-TECAIR (2-200 nM). f LNCaP cells, stably expressing wild-type (WT) ATG16L2-HA, HA-TECPR1, or their ATG5-binding mutants I18W and $1152 \mathrm{~W}$, respectively, were treated with $125 \mathrm{nM}$ Baf A1 for $8 \mathrm{~h}$ and immunoblotted for the indicated proteins. The ATG16L1 expression levels and LC3-II/LC3-I ratios from Baf A1-treated cells were quantified and graphed below (*, $p<0.01)$. g ATG16L2:ATG16L1 mRNA ratios were determined for multiple tumor types from TCGA datasets. $\mathbf{h}$ Lysates from wild-type, ATG16L1 KO, ATG16L2 KO, TECPR1 KO, ATG16L1/L2 DKO, ATG16L1/TECPR1 DKO, and ATG16L1/L2/TECPR1 TKO PC-3 cells were immunoblotted for subunits of the ATG12-ATG5-ATG16L1 complex (see also Supplementary Fig. S5b, c)

to the knockout of $A T G 16 L 1$ alone, although it did not eliminate it entirely (Fig. 5h, lanes 2 and 7).

Since the ATG16L1-binding pocket of ATG5 must be occupied to prevent ubiquitin conjugation and $\mathrm{PQC}$ of ATG5, ATG12, and ATG16L1, as observed with ATG16L1-binding mutants (Fig. 3), the residual ATG12-ATG5 conjugation in the triple-KO cells suggested that other unknown protein(s) were able to bind free ATG5 and partially compensate for the concurrent loss of ATG16L1, ATG16L2, and TECPR1. Therefore, the data indicate that ATG16L2, TECPR1, and likely other unknown ATG5-binding protein(s), compete with ATG16L1 for binding to ATG5 and collectively function as dominant-negative inhibitors of ATG12-ATG5ATG16L1 complex formation. It is imperative to reiterate that disruption of the ATG16L1-binding pocket in tumors through somatic ATG5 mutations and/or alternative mRNA splicing, simultaneously prevents all proteins from binding to the ATG16L1-binding pocket, thus highlighting its critical importance in the regulation of the ATG5-conjugation switch, ATG12-ATG5-ATG16L1 complex formation and autophagy in tumors.

\section{Discussion}

This study was initiated following our discovery that DU145 PCa cells did not express ATG5 due to an ATG5 splice donor site mutation, which ultimately triggered PQC of orphaned ATG12 and ATG16L1 and inactivated autophagy. We then identified and characterized ATG5 splice variants and more than 50 structure-guided and somatic cancer mutations, which revealed that the mutually stabilizing ATG5-ATG16L1 interaction was an essential prerequisite for ATG12 conjugation and ATG12-ATG5-ATG16L1 complex formation. Due to the unique structure of ATG5 in which both the N- and Ctermini collectively form the ATG16L1-binding pocket, somatic mutations and alternative/aberrant mRNA splicing affecting either region of the protein triggered complete proteasomal degradation of ATG5, and consequently ATG12 and ATG16L1 (Figs. 1, 3; Supplementary
Table S1). Based on these findings we propose an updated model for ATG12-ATG5-ATG16L1 complex formation in which free, unbound ATG5, ATG12, and ATG16L1 are inherently highly unstable proteins that continuously undergo PQC until a transient, but essential, ATG5ATG16L1 interaction temporarily impairs ubiquitin conjugation of ATG5, thereby stabilizing both proteins and allowing for ATG12 conjugation to ATG5 (Fig. 6). This initial lower affinity interaction between ATG5 and ATG16L1 likely stabilizes both proteins by masking E3 ubiquitin ligase binding sites and/or target lysine residues. Regardless, once formed, the ATG12-ATG5 conjugate displays a significantly enhanced affinity for ATG16L1, resulting in the formation of more stable ATG12-ATG5ATG16L1 complexes. Therefore, by controlling the fate of ATG5, the competing ATG12 and ubiquitin conjugation reactions effectively function as a molecular "conjugation switch" that regulates autophagy by integrating PQC of individual subunits with ATG12-ATG5-ATG16L1 complex formation.

In addition to ATG16L1, we determined that ATG16L2 - and to a lesser extent TECPR1-can hijack this conjugation switch by competitively binding to the ATG16L1binding pocket of ATG5, displacing ATG16L1, and triggering its proteasomal degradation (Fig. 6). While the ATG12-ATG5-ATG16L2 complex has no known function, the ATG12-ATG5-TECPR1 complex reportedly mediates selective autophagy of bacterial pathogens (xenophagy) and participates in autophagosome-lysosome fusion $^{30,31}$. Thus, while these complexes may indeed have important downstream functions in autophagy, neither catalyzes LC3 lipidation, which allows them to function as endogenous dominant-negative inhibitors of ATG12-ATG5-ATG16L1 complex assembly and canonical autophagy.

The fact that ATG5 is selectively inactivated by somatic mutations and/or alternative mRNA splicing (Figs. 1 and 3 ), and that $A T G 16 L 2$ is transcriptionally overexpressed, relative to $A T G 16 L 1$, in multiple tumor types (Fig. $5 \mathrm{~g}$ ), suggests that the inhibition of autophagy is indeed 


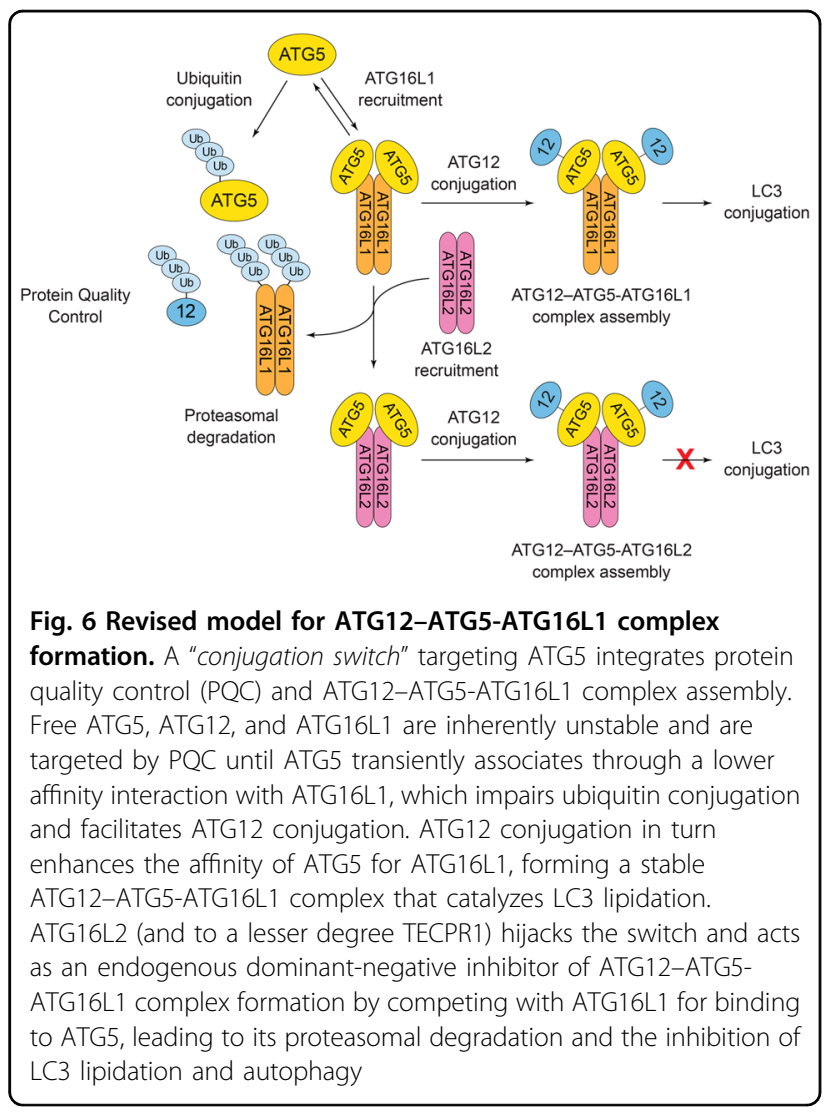

oncogenic. This is consistent with the current consensus that autophagy maintains homeostasis in pre-malignant cells and suppresses tumor initiation by eliminating injured mitochondria, oncogenic proteins and protein aggregates ${ }^{16,17}$. However, autophagy is also thought to be essential for tumor progression, metastasis and therapy resistance by promoting tumor cell survival during times of acute stress, e.g., in the hypoxic, oxidative, and nutrient-deprived tumor microenvironment prior to angiogenesis or in response to therapy ${ }^{32-34}$. Therefore, one would expect tumors possessing ATG5 loss-offunction mutations or overexpressing $A T G 16 L 2$ to be deficient in their abilities to progress, metastasize, and/or develop resistance to chemo- or radiotherapies. If true, small molecule "ATG16L2-mimetics" that target the critical ATG16L1-binding pocket of ATG5 and trigger PQC of ATG16L1 could represent a new class of highly selective autophagy inhibitors that could be used therapeutically for autophagy-proficient tumors, particularly those with low-ATG5 expression resulting from transcriptional repression, alternative mRNA splicing or hemizygous somatic mutations.

This possibility notwithstanding, the fact that DU145 $\mathrm{PCa}$ cells, which were originally isolated from a brain metastasis $^{35}$, are ATG5-deficient and readily form tumors in immunodeficient mice, suggests that ATG5 per se may not always be essential for tumor progression. The deletion of $\operatorname{Atg} 5$ or $\operatorname{Atg} 7$ has reportedly opposing effects on tumor progression, depending upon the status of $T p 53$ or Pten, in murine pancreatic tumor models ${ }^{36,37}$. Thus, inactivation of autophagy may actually be tumor promotive in some genetic contexts, possibly due to reduced autophagy-dependent tumor antigen presentation and tumor immunosurveillance ${ }^{38,39}$. It is also possible that, once initiated, certain human tumors evolve the ability to utilize an ATG5-independent form of autophagy that enables growth and progression. Evidence for ATG5independent autophagy has been reported in ATG5deficient cells in response to specific types of stress and during the induction of pluripotency ${ }^{40-43}$.

Another intriguing possibility is that ATG5 may possess tumor suppressive functions that are unrelated to autophagy so that the loss of ATG5 (or the subsequent degradation of ATG12 and ATG16L1 via PQC), confers a selective advantage to rapidly dividing or migrating tumor cells, despite the loss of canonical autophagy. ATG5 reportedly mediates apoptosis and mitotic catastrophe through interactions with $\mathrm{BCL}-\mathrm{X}_{\mathrm{L}}$ and survivin, respectively $^{44,45}$. Recently, unconjugated ATG12, which can induce apoptosis by binding to and inactivating antiapoptotic BCL-2 family members ${ }^{46}$, was shown to be downregulated through ubiquitination and proteasomal degradation in cancer cells possessing RAS mutations ${ }^{47,48}$. While it is unknown if ATG16L1 likewise possesses proapoptotic activity, hemizygous ATG5 deletions and ATG5 mRNA downregulation in human melanomas are associated with metastasis and poor patient survival ${ }^{49,50}$. Thus, in order to effectively evaluate the therapeutic potential of targeting the ATG16L1-binding pocket in ATG5, further investigation into the potential ATG5-independent forms of autophagy; the nonautophagic functions of ATG5, ATG12, and ATG16L1; and the impact of ATG5 inactivation on tumor progression in different tissues and genetic backgrounds will be required.

\section{Materials and methods}

\section{Reagents}

\begin{tabular}{|c|c|c|}
\hline Reagent & Source & Identifier \\
\hline \multicolumn{3}{|l|}{ Antibodies } \\
\hline AMBRA1 (1:500 dilution) & Cell Signaling Technology & Cat\#12250; RRID: N/A \\
\hline ATG3 (1:500 dilution) & Sigma-Aldrich & $\begin{array}{l}\text { Cat\#3415; RRID: } \\
\text { AB_2059244 }\end{array}$ \\
\hline $\begin{array}{l}\text { ATG5 (C-terminal) (1:500 } \\
\text { dilution) }\end{array}$ & Cell Signaling Technology & $\begin{array}{l}\text { Cat\#2630; RRID: } \\
\text { AB_2062340 }\end{array}$ \\
\hline $\begin{array}{l}\text { ATG5 (N-terminal) (1:8000 } \\
\text { dilution) }\end{array}$ & Novus Biologicals & $\begin{array}{l}\text { Cat\#NB110-53818; } \\
\text { RRID: AB_828587 }\end{array}$ \\
\hline ATG7 (1:500 dilution) & Cell Signaling Technology & $\begin{array}{l}\text { Cat\#2631; RRID: } \\
\text { AB_2227783 }\end{array}$ \\
\hline $\begin{array}{l}\text { ATG12 (D88H11) (1:500 } \\
\text { dilution) }\end{array}$ & Cell Signaling Technology & $\begin{array}{l}\text { Cat\#4180; RRID: } \\
\text { AB_1903898 }\end{array}$ \\
\hline ATG13 (1:2000 dilution) & Cell Signaling Technology & Cat\#6940; RRID: N/A \\
\hline
\end{tabular}




\begin{tabular}{|c|c|c|}
\hline Reagent & Source & Identifier \\
\hline $\begin{array}{l}\text { ATG16L1 (D6D5) (1:2000 } \\
\text { dilution) }\end{array}$ & Cell Signaling Technology & $\begin{array}{l}\text { Cat\#8089; RRID: } \\
\text { AB_10950320 }\end{array}$ \\
\hline Beclin 1 (1:1000 dilution) & Cell Signaling Technology & $\begin{array}{l}\text { Cat\#3738; RRID: } \\
\text { AB_490837 }\end{array}$ \\
\hline $\begin{array}{l}\beta \text {-Tubulin (1:20,000 } \\
\text { dilution) }\end{array}$ & $\begin{array}{l}\text { Developmental Studies } \\
\text { Hybridoma Bank (DSHB) }\end{array}$ & Cat\#E7-c; RRID: N/A \\
\hline $\begin{array}{l}\text { FIP200/RB1CC1 (1:4000 } \\
\text { dilution) }\end{array}$ & Proteintech Group & $\begin{array}{l}\text { Cat\#17250-1-AP; RRID } \\
\text { AB_10666428 }\end{array}$ \\
\hline $\begin{array}{l}\text { FLAG (M2) }(1: 32,000 \\
\text { dilution) }\end{array}$ & Sigma-Aldrich & $\begin{array}{l}\text { Cat\#F7425; RRID: } \\
\text { AB_439687 }\end{array}$ \\
\hline $\begin{array}{l}\text { GST (91G1) (1:20,000 } \\
\text { dilution) }\end{array}$ & Cell Signaling Technology & $\begin{array}{l}\text { Cat\#2625; RRID: } \\
\text { AB_490796 }\end{array}$ \\
\hline HA (1:8000 dilution) & Novus Biologicals & $\begin{array}{l}\text { Cat\#NB600-363; RRID: } \\
\text { AB_10001504 }\end{array}$ \\
\hline $\begin{array}{l}\text { IgG-peroxidase (mouse; } \\
\text { 1:2000 dilution) }\end{array}$ & Sigma-Aldrich & $\begin{array}{l}\text { Cat\#A4416; RRID: } \\
\text { AB_258167 }\end{array}$ \\
\hline $\begin{array}{l}\text { IgG-peroxidase (rabbit; } \\
1: 2,0000 \text { dilution) }\end{array}$ & Sigma-Aldrich & $\begin{array}{l}\text { Cat\#A4914; RRID: } \\
\text { AB_258207 }\end{array}$ \\
\hline LC3B (1:2000 dilution) & Cell Signaling Technology & $\begin{array}{l}\text { Cat\#2775; RRID: } \\
\text { AB_915950 }\end{array}$ \\
\hline MCL-1 (1:1000 dilution) & Cell Signaling Technology & $\begin{array}{l}\text { Cat\#4572; RRID: } \\
\text { AB_2281980 }\end{array}$ \\
\hline $\begin{array}{l}\text { PIK3C3/Nps34 (ZMD.350) } \\
\text { (1:1000 dilution) }\end{array}$ & Innovative Research & $\begin{array}{l}\text { Cat\#38-2100; RRID: } \\
\text { AB_431499 }\end{array}$ \\
\hline $\begin{array}{l}\text { p62/SQSTM1 (1:2000 } \\
\text { dilution) }\end{array}$ & Cell Signaling Technology & $\begin{array}{l}\text { Cat\#5114; RRID: } \\
\text { AB_10624872 }\end{array}$ \\
\hline ULK1 (1:500 dilution) & Cell Signaling Technology & $\begin{array}{l}\text { Cat\#8054 RRID: } \\
\text { AB_11178668 }\end{array}$ \\
\hline \multicolumn{3}{|l|}{ Chemicals } \\
\hline Bafilomycin A1 & Tocris Bioscience & $\begin{array}{l}\text { Cat\#1334; CAS: } 88899- \\
55-2\end{array}$ \\
\hline Cyclohexamide & Calbiochem & $\begin{array}{l}\text { Cat\#239763; CAS: 66- } \\
81-9\end{array}$ \\
\hline MG132 & Selleck Chemicals & $\begin{array}{l}\text { Cat\#S2619; CAS: } \\
133407-82-6\end{array}$ \\
\hline \multicolumn{3}{|l|}{ Oligonucleotides } \\
\hline RT-PCR primer sequences & This paper & See Table S2 \\
\hline $\begin{array}{l}\text { Genomic PCR primer } \\
\text { sequences }\end{array}$ & This paper & See Table S3 \\
\hline sgRNA oligo sequences & This paper & See Table S4 \\
\hline $\begin{array}{l}\text { ssODN donor template } \\
\text { sequence }\end{array}$ & This paper & See Table S5 \\
\hline Cloning primer sequences & This paper & See Table S6 \\
\hline $\begin{array}{l}\text { Site-directed mutagenesis } \\
\text { primer sequences }\end{array}$ & This paper & See Table S7 \\
\hline
\end{tabular}

\section{Expression constructs}

The pLOC lentiviral expression vector was obtained from the University of Texas MD Anderson Cancer Center (UTMDACC) Functional Genomics Core (FGC). FLAG-tag and HA-tag sequences were cloned into the BamHI/NheI of pLOC to generate an N-terminal FLAGtag (pLOC-NFLAG) or HA-tag (pLOC-NHA) vectors. HA-tag was also cloned into the NheI/AscI sites of pLOC to generate a C-terminal HA-tag (pLOC-HA). cDNA of ubiquitin was kindly provided by Dr. Colin Duckett (Duke University School of Medicine, Durham, NC). Ubiquitin was cloned into NheI/AscI sites of pLOC-NFLAG. cDNA for human ATG5 (Cat\#PLOHS_10007) was obtained from UTMDACC FGC (Houston, TX). Full-length ATG5 was cloned into BamHI/NheI sites of untagged pLOC and pLOC-HA. For alternative splice variant ATG5v3, only the predicted coding sequence was amplified by PCR and cloned into BamHI/NheI sites of pLOC-HA. ATG5v4 was generated by site-directed mutagenesis. ATG5v5, v6 and v7 splice variants were originally cloned into pGEM-T easy following reverse transcription polymerase chain reaction (RT-PCR) of mRNA extracted from DU145 and PC-3 PCa cells, and were then subcloned into BamHI/ NheI sites of pLOC-HA. For bacterial expression, fulllength $A T G 5$ was cloned into BamHI/NotI of malE-pET, a bacterial expression vector kindly provided by Dr. Hungwen (Ben) Liu (University of Texas at Austin, Austin, TX) that was derived from pET28b and contains the malE gene encoding a Maltose-binding protein (MBP) tag. All ATG5 mutations were introduced by site-directed mutagenesis, except for mutations predicted to cause alternative translation initiation and $\mathrm{N}$-terminal deletions (p. M1_V11del and p.M1_V59del) in which case only the predicted coding sequences were cloned. cDNA for murine Atg5 was a gift from Roberta Gottlieb (Addgene plasmid $\# 13095)^{51}$. Murine Atg5 was cloned into BamHI/ NheI sites of untagged pLOC.

To clone ATG16L1, cDNA was obtained from the UTMDACC FGC (Cat\#PLOHS_10007). The internal BamHI site of ATG16L1 was first removed by site-directed mutagenesis. Full-length and N-terminally deleted $(\Delta \mathrm{N})$ ATG16L1 (nucleotides 118-1824) were then amplified by PCR and cloned into the BamHI/NheI sites of pLOC-HA. cDNAs for ATG16L2 (Cat\#HsCD00342734) and TECPR1 (Cat\#HsCD00337885) were obtained from the Dana-Farber/Harvard Cancer Center DNA Resource Core. ATG16L2 and TECPR1 were cloned into the BamHI/NheI sites of pLOC-HA and the NheI/AscI sites of pLOC-NHA, respectively. Mutations of $A T G 16 L 2$ and TECPR1 were subsequently introduced by site-directed mutagenesis. The 16L1N-ATG5 fusion construct was created by cloning the ATG5-binding region of ATG16L1 (nucleotides 31-108) into the BamHI/NheI sites of pLOC, and a 15 base-pair GSlinker-ATG5 into the NheI/AscI sites. Mutations were once again introduced by site-directed mutagenesis. For bacterial expression vectors, nucleotides 1-207 of ATG16L1 (ATG16L1N) and ATG16L2 (ATG16L2N), as well as nucleotides 1696-1830 of TECPR1 (TECAIR), were cloned into the BamHI/NotI sites of pGEX-4T-1 (GE Healthcare Bio-Sciences \#28-9545-49).

\section{Cell lines and culture conditions}

DU145 (HTB-81), LNCaP clone FGC (CRL-1740), and PC-3 (CRL-1435) PCa cells were purchased from American Type Culture Collection (ATCC) and grown in RPMI-1640, supplemented with $10 \%$ fetal bovine serum (FBS) and $2 \mathrm{mM}$ L-glutamine, at $37^{\circ} \mathrm{C}$ in humidified air containing $5 \% \mathrm{CO}_{2}$ and were routinely passaged every 
3 days. $\operatorname{Atg} 5^{+/+}, \operatorname{Atg} 5^{-/-}, \operatorname{Atg} 7^{+/+}, \operatorname{Atg} 7^{--}, \operatorname{Atg} 12^{+/+}$, Atg12 $2^{-/-}$, Atg16L1 $1^{+/+}$, and Atg16l1 ${ }^{\Delta / \Delta}$ MEFs were kindly provided by Dr. Noburu Mizushima (University of Tokyo, Tokyo, JP) ${ }^{52}$, Dr. Masaaki Komatsu (Tokyo Metropolitan Institute of Medical Science, Tokyo, JP) ${ }^{53}$, Dr. Jayanta Debnath (University of California-San Francisco, San Francisco, CA) ${ }^{54}$, and Dr. Shizuo Akira (Osaka University, Osaka, JP) ${ }^{55}$, respectively. All MEFs, as well as human embryonic kidney cells (HEK293T) provided by Dr. Casey Wright (The University of Texas Medical Branch, Galveston, TX), were cultured in DMEM, supplemented with $10 \% \mathrm{FBS}$ and $4 \mathrm{mM}$ L-glutamine, at $32.5-37^{\circ} \mathrm{C}$ in humidified air containing $5 \% \mathrm{CO}_{2}$ and were routinely passaged every 3 days.

\section{RT-PCR and genomic PCR}

mRNA was isolated from LNCaP, PC-3, and DU145 $\mathrm{PCa}$ cells using an RNeasy Kit with on-column DNase digestion (Qiagen \#74104). cDNA was synthesized from $1 \mu \mathrm{g}$ of mRNA using SuperScript ${ }^{\mathrm{m}}$ III First-Strand Synthesis SuperMix for qRT-PCR (Invitrogen \#11752-050), and ATG5 was then amplified by PCR. Genomic DNA was isolated using the Wizard ${ }^{\oplus}$ Genomic DNA Purification Kit (Promega \#A1120). Genomic PCR was performed using FailSafe ${ }^{\mathrm{m}}$ PCR System (Lucigen \#FS99100). All PCR mixtures were resolved by gel electrophoresis and, when applicable, the resulting bands were cut, extracted and Sanger sequenced.

\section{Lentiviral transduction and stable cell line generation}

HEK293T cells were co-transfected with pLOC along with psPAX2 and pHCMV-G lentivirus packaging plasmids. psPAX2 was a gift from Dr. Didier Trono (Addgene plasmid \#12260). Approximately, $48 \mathrm{~h}$ following transfection, the medium was collected and $6 \mu \mathrm{L}$ of sterile hexadimethrine bromide $(5 \mu \mathrm{g} / \mu \mathrm{L} ; \quad$ Sigma-Aldrich \#H9268) was added. The collected medium was then filtered through a $0.45 \mu \mathrm{m}$ polyvinylidene fluoride (PVDF) syringe filter and incubated with target cells overnight. Transduction efficiency was evaluated by GFP expression and/or immunoblotting.

\section{CRISPR/Cas9 knockout and knock-in cell line generation}

For knockout cell lines, DNA oligonucleotides (oligos) containing gRNA target sequences for ATG5, ATG7, ATG16L1, ATG16L2, and TECPR1 were designed using the CRISPR Design Tool (http://crispr.mit.edu). DNA oligos were annealed and ligated into the lentiCRISPRv2 vector, a gift from Dr. Feng Zhang (Addgene plasmid \#52961), using an established protocol ${ }^{56}$. DU145, PC-3, or LNCaP PCa cells were then transduced as described above, and sorted into single cell clones by flow cytometery (BD Biosciences, FACSAria ${ }^{m}$ Fusion). The clones were screened for successful gene knockout by immunoblotting for the targeted protein, as well as for LC3B lipidation and p62 degradation. For ATG16L2 and TECPR1, no commercial antibodies were found to be suitable, therefore, all clones were screened by Sanger sequencing and analyzed using the ICE Analysis Tool (https://ice.synthego.com). As cancer cells are frequently polyploidal, CRISPR/Cas9-mediated insertions or deletions (indels) result in multiple unique alleles that make Sanger sequencing challenging. This tool uses an algorithm to deconvolute Sanger trace data into unique alleles, which are then given "contribution percentages" based on the relative heights of trace data. The ICE score refers to the percentage of total alleles possessing indels, while the KO score refers to the percentage of total alleles possessing indels predicted to induce frameshifts and loss of gene function (see https://www.biorxiv.org/content/ biorxiv/early/2019/01/14/251082.full.pdf for details). Clones with $>90 \% \mathrm{KO}$ scores were considered true knock outs.

For the ATG5 splice donor site knock-in cell line, DNA oligos containing a gRNA target sequence for exon 6 of ATG5 were designed using the CRISPR Design Tool. DNA oligos were annealed and ligated into the lentiCRISPRv2 vector. A single-strand DNA oligonucleotide $(\mathrm{ssODN})$ donor template was designed to introduce the desired splice donor site mutation $($ c. $573+1 \mathrm{~A}>\mathrm{G})$ and four additional silent mutations intended to prevent cleavage of the donor template. DU145 cells were transduced as described above and electroporated the following day with the ssODN donor template $(10 \mu \mathrm{M})$ using the

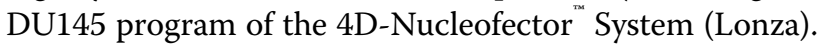
Cells were then sorted into single clones by flow cytometry as described above. Successful homologous recombination was determined by immunoblotting and sequencing.

\section{Immunoblot analysis}

Cells were lysed in ice-cold radioimmunoprecipitation assay buffer (50 mM Tris- $\mathrm{HCl} \mathrm{pH} 7.4,150 \mathrm{mM} \mathrm{NaCl}, 1 \%$ NP-40, 1\% Na-deoxycholate) with protease inhibitors. Lysate protein concentrations were quantified using the Bradford assay, and $50 \mu \mathrm{g}$ of lysate was loaded into $10-15 \%$ polyacrylamide gels and separated at $125 \mathrm{~V}$ for $1.5 \mathrm{~h}$. The resolved proteins were then transferred onto nitrocellulose membranes (or PVDF membranes for ATG12 and LC3B immunoblots) for $2 \mathrm{~h}$ at $90 \mathrm{~V}$. Membranes were blocked in $5 \%$ nonfat milk in TBS-T $(50 \mathrm{mM}$ Tris, $150 \mathrm{mM} \mathrm{NaCl}, 0.1 \%$ Tween-20, $\mathrm{pH}$ 7.6) for $1 \mathrm{~h}$ at room temperature, washed twice in TBS-T for $5 \mathrm{~min}$, and incubated in primary antibody overnight at $4{ }^{\circ} \mathrm{C}$ with constant agitation. Membranes were then washed and incubated for $1 \mathrm{~h}$ at room temperature with mouse or rabbit IgG-HRP secondary antibodies (Sigma-Aldrich \#A4416 and \#A4914) diluted 1:2000 with 5\% milk in TBS- 
T. Finally, membranes were washed and developed using enhanced chemiluminescence (PerkinElmer \#NEL104001). When applicable, densitometry was performed using Image Studio Lite (LI-COR Biosciences).

\section{Recombinant protein expression and GST pull downs}

All forms of recombinant MBP-ATG5, as well as GST, GST-ATG16L1N, GST-ATG16L2N, and GST-TECAIR were expressed in Escherichia coli strain BL21(DE3)pLysS (EMD Millipore \#694510) following an overnight induction at $18^{\circ} \mathrm{C}$ with $1 \mathrm{mM}$ isopropyl- $\beta$-D-thiogalactoside (IPTG). Recombinant proteins were then purified using fast protein liquid chromatography, coupled to a $\mathrm{Ni}^{2+}{ }_{-} \mathrm{NTA}$ column (Thermo Scientific \#88222) or a glutathione column (EMD Millipore \#70541). All proteins were dialyzed into phosphate-buffered saline (PBS) and concentrations were determined using the Bradford assay. For GST pulldown assays of recombinant MBP-ATG5 mutants, $200 \mathrm{nM}$ GST-ATG16L1N was incubated with glutathione resin overnight at $4{ }^{\circ} \mathrm{C}$ with constant mixing in GST pulldown lysis buffer $(20 \mathrm{mM}$ Tris- $\mathrm{HCl} \mathrm{pH} 8.0,200 \mathrm{mM}$ $\mathrm{NaCl}, 1 \mathrm{mM}$ EDTA, 0.5\% NP-40). Resin was washed 3 times with lysis buffer and incubated with $15 \mu \mathrm{g}$ of wildtype or mutant MBP-ATG5 for $1 \mathrm{~h}$. Resin was washed 3 times again and proteins were eluted with $30 \mathrm{mM}$ reduced glutathione for $30 \mathrm{~min}$. For GST pull-down assays from wild-type and ATG7-knockout PC-3 cell lysates, cells were lysed in ice-cold GST pull-down lysis buffer with protease inhibitors. Lysate concentrations were measured using the Bradford assay, and wild-type and ATG7-KO lysates were mixed in a 1:3.5 ratio to equalize the levels of conjugated and unconjugated ATG5. Pull downs were performed as described above with $1.5 \mathrm{mg}$ total lysate. Samples were then evaluated by immunoblotting using antibodies to GST and ATG5.

\section{FLAG-ubiquitin immunoprecipitation}

DU145 cells stably expressing ATG5 \pm FLAG-ubiquitin were treated with $10 \mu \mathrm{M}$ MG132 for $8 \mathrm{~h}$ and lysed in icecold Triton X-100 lysis buffer (50 mM Tris-HCl, pH 7.4, $150 \mathrm{mM} \mathrm{NaCl}, 1 \mathrm{mM}$ EDTA and 1\% Triton X-100). Lysate concentrations were normalized using the Bradford assay and incubated at $4{ }^{\circ} \mathrm{C}$ overnight with $80 \mu \mathrm{L}$ of ANTIFLAG M2 Affinity Gel (Sigma-Aldrich \#A2220) with constant agitation. Resin was washed three times with icecold PBS and boiled in nonreducing Laemmli sample buffer (125 nM Tris-HCl, pH 6.8, 20\% glycerol, 4\% SDS, $0.1 \%$ bromophenol blue). Samples were then evaluated by immunoblotting using antibodies to FLAG and ATG5.

\section{Bioinformatic analyses}

Rendering of the human ATG12 (aa 52-140)-ATG5ATG16L1N (aa 11-43) (PDB ID: 4GDL) and ATG5ATG16L1N (PDB ID: 4TQ0) crystal structures, as well as modeling of the ATG16L2N peptide, was performed with the UCSF Chimera package (https://www.cgl.ucsf.edu/ chimera//) ${ }^{27,28,57}$. All alternative ATG5 splice variants and their predicted transcripts were taken from the National Center for Biotechnology Information (NCBI) RefSeq database (https://www.ncbi.nlm.noh.gov/refseq/) ${ }^{25}$. Somatic ATG5 mutations were collected from the Catalog of Somatic Mutations in Cancer (COSMIC: http://cancer. sanger.ac.uk/cosmic), the International Cancer Genome Consortium (ICGC: https://icgc.org), the Broad Institute Cancer Cell Line Encyclopedia (CCLE: https://portals. broadinstitute.org/ccle), and cBioPortal for Cancer Genomics (http://www.cbioportal.org) databases ${ }^{58-62}$. Somatic mutations from tumor samples were verified with Annotated Somatic Mutation Variant Cell Format (VCF) files associated with each de-identified donor. The effects of ATG5 splice site mutations on mRNA splicing were predicted, in silico, using the Human Splicing Finder (http:// www.umd.be/HSF/) ${ }^{24}$. This algorithm uses position weight matrices to calculate consensus values (CVs), which are the sum of the weighted scores given to each nucleotide based on its position within the conserved splicing motif. The CV variation \% refers to the difference in CVs between the wild-type and mutant sequences as a percentage of the wild-type CV.

TCGA level 3 data were downloaded from the TCGA data portal (https://portal.gdc.cancer.gov) and clinical information was extracted. Datasets with $<25$ normal samples were excluded from the analysis. ATG5 splice variants $v 1$ (uc003prf), v3(uc003prf.2), v4(uc003prg.2), and $v 5$ (uc010kdb.2) were identified in the TCGA datasets; however, $v 4$ and $v 5$ were not expressed in the vast majority of samples. Therefore, the percentage of full-length ATG5 mRNA (ATG5v1; uc003prf) expression was calculated from the sum of ATG5 $(v 1+v 3)$ mRNA expression in normal and tumor tissue and plotted using R. The ATG16L2: ATG16L1 mRNA expression ratio from normal and tumor tissue was also calculated and plotted using $\mathrm{R}$.

\section{Statistical analyses}

All experiments were performed at least three times with each Western blot serving as a representative image. In some experiments, densitometry for bands corresponding to ATG12-ATG5, ATG16L1, and LC3 blots was performed and statistical significance determined using ANOVA with a Student-Newman-Kuels post hoc analysis. For mRNA expression comparisons between tumor and normal tissue from TCGA datasets, unpaired two-tailed $t$ tests were performed using $\mathrm{R}$. In all cases $p<$ 0.01 was considered statistically significant.

\section{Data availability}

The authors can confirm that all relevant data are included in the paper and/or its Supplementary files. 


\section{Acknowledgements}

The authors wish to thank Drs. Noburu Mizushima (University of Tokyo, JP), Masaaki Komatsu (Tokyo Metropolitan Institute of Medical Science, JP), Jayanta Debnath (University of California, San Francisco, USA) and Shizou Akira (Osaka University, JP) for providing $\operatorname{Atg} 5^{-1-}, \operatorname{Atg}^{-1-}, \operatorname{Atg} 12^{-1-}$, and Atg $1611^{1 / \Delta} \mathrm{MEFs}$, respectively ${ }^{52-55}$. This work was supported by an NIEHS Toxicology Training Grant fellowship (T32ESO7274) to D.J.W.; a DOD grant (W81XWH-16-1-0575) to D.G.T.; and NCI/NIGMS grants (CA129521 and GM096101) and an Institutional Research Grant (IRG) from The University of Texas MD Anderson Cancer Center (UTMDACC) to S.B.B. This study utilized the UTMDACC Protein Array and Analysis Core (PAAC) Facility (CPRIT PR130432), as well as the UTMDACC Flow Cytometry and Cellular Imaging Core Facility (FCCICF)-Smithville and the UTMDACC Functional Genomics Core (FGC) Facility, which are supported by an NIH/NCl Cancer Center Support Grant (P30 CA016672).

\section{Author details}

'Department of Epigenetics and Molecular Carcinogenesis, The University of Texas MD Anderson Cancer Center, Smithville, TX 78957, USA. ${ }^{2}$ Department of Pharmacology and Therapeutics, Roswell Park Cancer Institute, Buffalo, NY 14263, USA. ${ }^{3}$ Cancer Stem Cell Institute, Research Center for Translational Medicine, East Hospital, Tongji University School of Medicine, Shanghai 200120, China

\section{Author contributions}

D.J.W. and S.B.B. conceptualized and designed the experiments. H.P.C. performed the bioinformatic analyses. D.J.W. carried out all the remaining experiments. D.G.T. provided key reagents and expertise. D.J.W. and S.B.B. analyzed the data and wrote the paper. All authors discussed the results and commented on the paper.

\section{Competing interests}

The authors declare no competing interests.

\section{Publisher's note}

Springer Nature remains neutral with regard to jurisdictional claims in published maps and institutional affiliations.

Supplementary Information accompanies the paper at (https://doi.org/ 10.1038/s41421-019-0110-1).

Received: 8 October 2018 Revised: 26 June 2019 Accepted: 30 June 2019 Published online: 27 August 2019

\section{References}

1. Shibutani, S. T. \& Yoshimori, T. A current perspective of autophagosome biogenesis. Cell Res. 24, 58-68 (2014).

2. Geng, J. \& Klionsky, D. J. The Atg8 and Atg12 ubiquitin-like conjugation systems in macroautophagy. 'Protein modifications: beyond the usual suspects' review series. EMBO Rep. 9, 859-864 (2008).

3. Noda, N. N. \& Inagaki, F. Mechanisms of Autophagy. Annu Rev. Biophys. 44 101-122 (2015).

4. Yamaguchi, M. et al. Structural insights into Atg10-mediated formation of the autophagy-essential Atg12-Atg5 conjugate. Structure 20, 1244-1254 (2012).

5. Dooley, H. C. et al. WIPI2 links LC3 conjugation with PI3P, autophagosome formation, and pathogen clearance by recruiting Atg12-5-16L1. Mol. Cell 55, 238-252 (2014).

6. Gammoh, N., Florey, O., Overholtzer, M. \& Jiang, X. Interaction between FIP200 and ATG16L1 distinguishes ULK1 complex-dependent and -independent autophagy. Nat. Struct. Mol. Biol. 20, 144-149 (2013).

7. Nishimura, T. et al. FIP200 regulates targeting of Atg16L1 to the isolation membrane. EMBO Rep. 14, 284-291 (2013).

8. Metlagel, Z., Otomo, C., Takaesu, G. \& Otomo, T. Structural basis of ATG3 recognition by the autophagic ubiquitin-like protein ATG12. Proc. Natl Acad. Sci. USA 110, 18844-18849 (2013).

9. Nath, S. et al. Lipidation of the LC3/GABARAP family of autophagy proteins relies on a membrane-curvature-sensing domain in Atg3. Nat. Cell Biol. 16, 415-424 (2014).
10. Noda, N. N., Fujioka, Y., Hanada, T., Ohsumi, Y. \& Inagaki, F. Structure of the Atg12-Atg5 conjugate reveals a platform for stimulating Atg8-PE conjugation. EMBO Rep. 14, 206-211 (2013).

11. Romanov, J. et al. Mechanism and functions of membrane binding by the Atg5-Atg12/Atg16 complex during autophagosome formation. EMBO J. 31, 4304-4317 (2012)

12. Sakoh-Nakatogawa, M. et al. Atg12-Atg5 conjugate enhances E2 activity of Atg3 by rearranging its catalytic site. Nat. Struct. Mol. Biol. 20, 433-439 (2013).

13. Behrends, C., Sowa, M. E., Gygi, S. P. \& Harper, J. W. Network organization of the human autophagy system. Nature 466, 68-76 (2010).

14. Schaaf, M. B., Keulers, T. G., Vooijs, M. A. \& Rouschop, K. M. LC3/GABARAP family proteins: autophagy-(un)related functions. FASEB J. 30, 3961-3978 (2016).

15. Nguyen, T. N. et al. Atg8 family LC3/GABARAP proteins are crucial for autophagosome-lysosome fusion but not autophagosome formation during PINK1/Parkin mitophagy and starvation. J. Cell Biol. 215, 857-874 (2016).

16. Galluzzi, L. et al. Autophagy in malignant transformation and cancer progression. EMBO J. 34, 856-880 (2015).

17. Takamura, A. et al. Autophagy-deficient mice develop multiple liver tumors Genes Dev. 25, 795-800 (2011).

18. Amaravadi, R., Kimmelman, A. C. \& White, E. Recent insights into the function of autophagy in cancer. Genes Dev. 30, 1913-1930 (2016).

19. Levy, J. M. M., Towers, C. G. \& Thorburn, A. Targeting autophagy in cancer. Nat. Rev. Cancer 17, 528-542 (2017).

20. Lebovitz, C. B. et al. Cross-cancer profiling of molecular alterations within the human autophagy interaction network. Autophagy 11, 1668-1687 (2015).

21. Korolchuk, V. I., Menzies, F. M. \& Rubinsztein, D. C. Mechanisms of cross-talk between the ubiquitin-proteasome and autophagy-lysosome systems. FEBS Lett. 584, 1393-1398 (2010).

22. Ouyang, D. Y. et al. Autophagy is differentially induced in prostate cancer LNCaP, DU145 and PC-3 cells via distinct splicing profiles of ATG5. Autophagy 9. 20-32 (2013)

23. Gao, D. et al. Organoid cultures derived from patients with advanced prostate cancer. Cell 159, 176-187 (2014).

24. Desmet, F. O. et al. Human Splicing Finder: an online bioinformatics tool to predict splicing signals. Nucleic Acids Res. 37, e67 (2009).

25. Pruitt, K. D. et al. RefSeq: an update on mammalian reference sequences Nucleic Acids Res. 42, D756-D763 (2014).

26. Sveen, A., Kilpinen, S., Ruusulehto, A., Lothe, R. A. \& Skotheim, R. I. Aberrant RNA splicing in cancer; expression changes and driver mutations of splicing factor genes. Oncogene 35, 2413-2427 (2016).

27. Otomo, C., Metlagel, Z., Takaesu, G. \& Otomo, T. Structure of the human ATG12 ATG5 conjugate required for LC3 lipidation in autophagy. Nat. Struct. Mol. Biol. 20, 59-66 (2013).

28. Kim, J. H. et al. Insights into autophagosome maturation revealed by the structures of ATG5 with its interacting partners. Autophagy 11, 75-87 (2015).

29. Ishibashi, K. et al. Atg16L2, a novel isoform of mammalian Atg16L that is not essential for canonical autophagy despite forming an Atg12-5-16L2 complex. Autophagy 7, 1500-1513 (2011).

30. Ogawa, M. et al. A Tecpr1-dependent selective autophagy pathway targets bacterial pathogens. Cell Host Microbe 9, 376-389 (2011).

31. Chen, D. et al. A mammalian autophagosome maturation mechanism mediated by TECPR1 and the Atg12-Atg5 conjugate. Mol. Cell 45, 629-641 (2012).

32. Yang, $\mathrm{S}$. et al. Pancreatic cancers require autophagy for tumor growth. Genes Dev. 25, 717-729 (2011).

33. Amaravadi, R. K. et al. Autophagy inhibition enhances therapy-induced apoptosis in a Myc-induced model of lymphoma. J. Clin. Invest. 117, 326-336 (2007).

34. Santanam, $U$. et al. Atg7 cooperates with Pten loss to drive prostate cancer tumor growth. Genes Dev. 30, 399-407 (2016).

35. Stone, K. R., Mickey, D. D., Wunderli, H., Mickey, G. H. \& Paulson, D. F. Isolation of a human prostate carcinoma cell line (DU 145). Int J. Cancer 21, 274-281 (1978).

36. Rosenfeldt, M. T. et al. p53 status determines the role of autophagy in pancreatic tumour development. Nature 504, 296-300 (2013).

37. Rosenfeldt, M. T. et al. PTEN deficiency permits the formation of pancreatic cancer in the absence of autophagy. Cell Death Differ. 24, 1303-1304 (2017).

38. Michaud, M. et al. Autophagy-dependent anticancer immune responses induced by chemotherapeutic agents in mice. Science $\mathbf{3 3 4}$ 1573-1577 (2011). 
39. Ko, A. et al. Autophagy inhibition radiosensitizes in vitro, yet reduces radioresponses in vivo due to deficient immunogenic signalling. Cell Death Differ. 21, 92-99 (2014).

40. Nishida, Y. et al. Discovery of Atg5/Atg7-independent alternative macroautophagy. Nature 461, 654-658 (2009).

41. Ra, E. A. et al. TRIM31 promotes Atg5/Atg7-independent autophagy in intestinal cells. Nat. Commun. 7, 11726 (2016).

42. Honda, S. et al. Ulk1-mediated Atg5-independent macroautophagy mediates elimination of mitochondria from embryonic reticulocytes. Nat. Commun. 5, 4004 (2014).

43. Ma, T. et al. Atg5-independent autophagy regulates mitochondrial clearance and is essential for iPSC reprogramming. Nat. Cell Biol. 17, 1379-1387 (2015).

44. Yousefi, S. et al. Calpain-mediated cleavage of Atg5 switches autophagy to apoptosis. Nat. Cell Biol. 8, 1124-1132 (2006).

45. Maskey, D. et al. ATG5 is induced by DNA-damaging agents and promotes mitotic catastrophe independent of autophagy. Nat. Commun. $\mathbf{4}$ 2130 (2013).

46. Rubinstein, A. D., Eisenstein, M., Ber, Y., Bialik, S. \& Kimchi, A. The autophagy protein Atg12 associates with antiapoptotic Bcl-2 family members to promote mitochondrial apoptosis. Mol. Cell 44, 698-709 (2011).

47. Haller, M. et al. Ubiquitination and proteasomal degradation of ATG12 regulates its proapoptotic activity. Autophagy 10, 2269-2278 (2014).

48. Yoo, B. H. et al. Oncogenic RAS-induced downregulation of ATG12 is required for survival of malignant intestinal epithelial cells. Autophagy 14, $1-18$ (2017).

49. Liu, H. et al. Down-regulation of autophagy-related protein 5 (ATG5) contributes to the pathogenesis of early-stage cutaneous melanoma. Sci. Transl. Med. 5, 202 ra123 (2013).
50. Garcia-Fernandez, M. et al. Metastatic risk and resistance to BRAF inhibitors in melanoma defined by selective allelic loss of ATG5. Autophagy 12, 1776-1790 (2016).

51. Hamacher-Brady, A. et al. Response to myocardial ischemia/reperfusion injury involves Bnip3 and autophagy. Cell Death Differ. 14, 146-157 (2007).

52. Kuma, A. et al. The role of autophagy during the early neonatal starvation period. Nature 432, 1032-1036 (2004).

53. Komatsu, M. et al. Impairment of starvation-induced and constitutive autophagy in Atg7-deficient mice. J. Cell Biol. 169, 425-434 (2005).

54. Malhotra, R., Warne, J. P., Salas, E., Xu, A. W. \& Debnath, J. Loss of Atg12, but not Atg5, in pro-opiomelanocortin neurons exacerbates diet-induced obesity. Autophagy 11, 145-154 (2015).

55. Saitoh, T. et al. Loss of the autophagy protein Atg16L1 enhances endotoxininduced IL-1beta production. Nature 456, 264-268 (2008).

56. Sanjana, N. E., Shalem, O. \& Zhang, F. Improved vectors and genome-wide libraries for CRISPR screening. Nat. Methods 11, 783-784 (2014).

57. Pettersen, E. F. et al. UCSF Chimera-a visualization system for exploratory research and analysis. J. Comput Chem. 25, 1605-1612 (2004).

58. Forbes, S. A. et al. COSMIC: exploring the world's knowledge of somatic mutations in human cancer. Nucleic Acids Res. 43, D805-D811 (2015).

59. International Cancer Genome, C. et al. International network of cancer genome projects. Nature 464, 993-998 (2010).

60. Cerami, E. et al. The CBio cancer genomics portal: an open platform for exploring multidimensional cancer genomics data. Cancer Discov. 2, 401-404 (2012).

61. Gao, J. et al. Integrative analysis of complex cancer genomics and clinical profiles using the cBioPortal. Sci. Signal 6, pl1 (2013).

62. Barretina, J. et al. The Cancer Cell Line Encyclopedia enables predictive modelling of anticancer drug sensitivity. Nature 483, 603-607 (2012). 